\title{
The flexible N-terminus of BchL protects its [4Fe-4S] cluster in
}

\section{oxygenic environments and autoinhibits activity}

Elliot Corless ${ }^{1}$, Syed Muhammad Saad Imran ${ }^{\sharp, 2}$, Maxwell B. Watkins ${ }^{\sharp 2,3}$, Sofia Origanti ${ }^{1,4}$, JohnPaul Bacik ${ }^{3}$, Robert Kitelinger ${ }^{1}$, Mark Soffe ${ }^{5}$, Karamatullah Danyal ${ }^{5}$, Lance C. Seefeldt ${ }^{5}$, Brian Bennett $^{6}$, Nozomi Ando ${ }^{2,3, *}$ and Edwin Antony ${ }^{1,7, *}$

${ }^{1}$ Department of Biological Sciences, Marquette University, Milwaukee, WI 53201

${ }^{2}$ Department of Chemistry, Princeton University, Princeton, NJ 08544

${ }^{3}$ Department of Chemistry and Chemical Biology, Cornell University, Ithaca, NY

${ }^{4}$ Department of Biology, Saint Louis University, St. Louis, MO

${ }^{5}$ Department of Chemistry and Biochemistry, Utah State University, Logan, UT 84322

${ }^{6}$ Department of Physics, Marquette University, Milwaukee, WI 53233

${ }^{7}$ Department of Biochemistry, Saint Louis University School of Medicine, St. Louis, MO 63104

Running Title: ATP usage in DPOR.

\# Equal author contributions

*Address correspondence to:

Edwin Antony, Department of Biochemistry and Molecular Biology, St. Louis University School of Medicine, 1205 Carr Lane, St. Louis, MO 63104. Email: edwin.antony@health.slu.edu, Ph: (314) 977 9257. Nozomi Ando, Department of Chemistry and Chemical Biology, Cornell University, Ithaca, NY 14853. Email: nozomi.ando@ cornell.edu, Ph: (607) 2559454 


\section{Abbreviations}

ET: electron transfer

DPOR: dark-operative protochlorophyllide oxidoreductase

Pchlide: protochlorophyllide

Chlide: chlorophyllide

\section{Keywords}

Electron Transfer, Nitrogenase, Nitrogenase-like enzymes, DPOR, Photosynthesis, Iron-Sulfur cluster 


\section{Abstract}

The dark-operative protochlorophyllide oxidoreductase (DPOR) enzyme contains two [4Fe-4S]containing component proteins (BchL and BchNB) that assemble in an ATP-dependent fashion to coordinate electron transfer and reduction of protochlorophyllide to chlorophyllide. Photosynthesis generates an oxygenic environment that is non-optimal for [Fe-S] clusters and we here present an elegant evolutionarily conserved mechanism in BchL to protect its [4Fe-4S] cluster. We present a crystal structure of BchL in the nucleotide-free form with an ordered $\mathrm{N}$-terminus that shields the [4Fe-4S] cluster at the docking interface between BchL and BchNB. Amino acid substitutions that perturb the shielding of the $[4 \mathrm{Fe}-4 \mathrm{~S}]$ cluster produce an unstable, but hyper-active enzyme complex, suggesting a role for the N-terminus in both auto-inhibition and enzyme stability. Upon ATP binding, a patch of amino acids, Asp-Phe-Asp ('DFD patch'), situated at the mouth of the BchL ATP-binding pocket promotes inter-subunit cross stabilization of the two subunits and relieves the auto-inhibition by the N-terminus. A linked BchL dimer with one defective ATP-binding site does not support substrate reduction, illustrating that nucleotide binding to both subunits is a prerequisite for the inter-subunit cross stabilization. We propose that ATP-binding produces a conformational compaction of the BchL homodimer leading to a release of the flexible $\mathrm{N}$-terminus from blocking the $[4 \mathrm{Fe}-4 \mathrm{~S}]$ cluster and promotes complex formation with BchNB to drive electron transfer. The auto-inhibitive feature and release mechanism appear unique to DPOR and is not found in the structurally similar nitrogenase. 


\section{Introduction}

Photosynthetic organisms utilize chlorophyll or bacteriochlorophyll to capture light for their energy requirements. The multi-step enzymatic biosynthesis of both these compounds are similar in the cell except for the penultimate reduction of protochlorophyllide (Pchlide) to produce chlorophyllide (Chlide). ${ }^{1,2}$ Angiosperms use a light-dependent protochlorophyllide oxidoreductase to catalyze the reduction, whereas gymnosperms, cyanobacteria, algae, bryophytes and pteridophytes possess a light-independent enzyme called dark-operative protochlorophyllide oxidoreductase (DPOR; Fig. 1a). ${ }^{3}$ Photosynthetic bacteria that are anoxygenic, such as Rhodobacter capsulatus, rely exclusively on the activity of DPOR for synthesis of bacteriochlorophyll. ${ }^{3}$ DPOR catalyzes the stereospecific reduction of the $\mathrm{C} 17=\mathrm{C} 18$ double bond of Pchlide to form Chlide (Fig. 1b). This reduction forms the conjugated $\pi$-system in the chlorin structure of chlorophyll-a which leads to a parental shift in the spectral properties required for photosynthesis. ${ }^{4,5}$

DPOR is structurally homologous to nitrogenase, the enzyme responsible for reducing dinitrogen to ammonia. DPOR is composed of two components: a homodimeric L-protein (BchL) and an heterotetrameric NB-protein $(\mathrm{BchNB}){ }^{5,6} \mathrm{BchL}$ serves as the ATP-dependent electron donor, and BchNB is the electron acceptor containing the active site for Pchlide binding and reduction (Fig. 1a). A multi-step reaction cycle has been proposed for DPOR function with the following overall reaction stoichiometry (Fig. 1b) ${ }^{4}$ :

$$
\text { Pchlide }+4 \mathrm{ATP}+2 \mathrm{e}^{-}+2 \mathrm{H}^{+}+4 \mathrm{H}_{2} \mathrm{O} \rightarrow \text { Chlide }+4 \mathrm{ADP}+4 \mathrm{P}_{\mathrm{i}}
$$

Give the structural similarity to nitrogenase, ATP binding to BchL is thought to promote its transient association with BchNB followed by a single electron transfer (ET) to Pchlide. ATP hydrolysis drives the dissociation of the protein complex. Two such rounds of ATP-dependent ET are 
necessary for Pchlide reduction, and the minimum stoichiometry of ATP/molecule of Chlide formed has been determined to be $4 .^{7}$ However, the details of how ATP binding promotes complex formation between $\mathrm{BchL}$ and BchNB are poorly resolved.

High-resolution crystal structures of the BchL dimer complexed with ADP (PDB:3FWY) ${ }^{8}$, and stabilized in a higher-order complex with the BchNB tetramer by $\mathrm{ADP}-\mathrm{AlF}_{3}$ (PDB:2YNM; Fig. 1a) ${ }^{9}$ provide several key molecular insights: BchL contains one [4Fe-4S] cluster ligated by 2 conserved cysteine residues from each subunit of the dimer. The subunits of BchL each have an active site for ATP, including conserved binding (Walker A) and hydrolysis (Walker B) motifs. Each half of the BchNB tetramer contains a substrate (Pchlide) binding site and one [4Fe-4S] cluster which functions as the electron acceptor from BchL (Fig. 1a). This cluster is ligated by 3 cysteine residues from $\mathrm{BchN}$ and one uncommon aspartic acid ligand from $\mathrm{BchB}$. BchL sits across the top of BchNB, placing their metal clusters in relatively close proximity ( $16 \AA$; Fig. 1a) ${ }^{9}$. Thus, ATP binding is hypothesized to drive formation of the complex. ATP hydrolysis has been proposed to drive electron transfer. In the homologous nitrogenase system, ATP hydrolysis occurs post-ET suggesting that hydrolysis likely drives complex dissociation post-ET. ${ }^{10}$ Given the structural similarities between DPOR and nitrogenase, we hypothesize ATP hydrolysis is also likely to promote complex dissociation in DPOR. Here, we address three key questions about ATP usage by BchL: How does binding of 2 ATP molecules collectively transmit information from the ATP binding sites to the $[4 \mathrm{Fe}-4 \mathrm{~S}]$ cluster of $\mathrm{BchL}$ along with the interface where it complexes with BchNB? What role does ATP play in electron transfer? Are both ATP binding events necessary?

We present a crystal structure of Rhodobacter sphaeroides BchL in the nucleotide-free state. This structure reveals novel electron density for a flexible $\mathrm{N}$-terminal region that is bound across the face of the BchL $[4 \mathrm{Fe}-4 \mathrm{~S}]$ cluster, suggesting a potential regulatory role. We show that 
amino acid substitutions within this flexible $\mathrm{N}$-terminal region enhance the kinetics of substrate reduction, pointing to a functionally suppressive role for this interaction. Additionally, inter-subunit contacts between BchL and the bound-ATP are critical for substrate reduction activity. Finally, we show that ATP binding to both subunits is required to promote conformational changes requisite for reduction of Pchlide to Chlide. We propose a model where ATP-driven cross stabilization of the homodimer promotes the release of the flexible $\mathrm{N}$-terminus and drives formation of the DPOR complex towards ET and substrate reduction.

\section{Results}

Crystal structure of nucleotide-free BchL suggests regulation through a redox switch and a flexible $N$-terminal region

BchL was crystallized anaerobically in the absence of nucleotides, and a crystal structure was determined to a resolution of $2.6 \AA$ (Supplemental Table 1). The asymmetric unit contains four BchL chains: chains A and B form one BchL dimer, and chains C and D comprise the other (Supplemental Fig. 1a,b). The overall structure of BchL is similar to previously published crystal structures (Fig. 2a-c), but in this nucleotide-free state, the top face of the dimer is more open compared

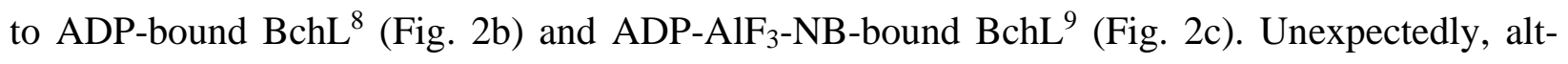
hough the N-terminus is disordered and not observed in all previous BchL structures (residues 129 in 3FWY and residues 1-27 in 2YNM from $P$. marinus), we observe clear electron density at the $\mathrm{N}$-terminus of chain $\mathrm{C}$ in our nucleotide-free structure, which we were able to model as residues 16 to 29 (Supplemental Fig. 1b). Interestingly, the flexible N-terminal region is observed bound across the $[4 \mathrm{Fe}-4 \mathrm{~S}]$ cluster in our structure (Fig. 2a) covering a surface that is normally used to 
interface with BchNB (Fig. 2c). In the chain C/D dimer, Asp23 of the N-terminal region interacts with Gln168, Gly16 forms an H-bond to Cys126 (an [4Fe-4S] cluster ligating residue) of the opposing chain, while Ser17 interacts with Gly161 of chain C via the backbone of G125 in chain D (Fig. 2d, Supplemental Fig. 1c). Based on the structure of the ADP-AlF 3 -stabilized DPOR complex (PDB: $2 \mathrm{NYM})^{9}$, the residues corresponding to Cys126, Gly161, and Gln168 in BchL $(R$. sphaeroides numbering) are three of the twelve residues that interact with BchNB during the formation of the active complex, as predicted by PDBePISA interface analysis ${ }^{11}$ (Fig. 2c, purple surface). Notably, the flexible N-terminal protective region (residues 1-29) is only conserved among DPOR BchL-proteins and is not observed in other homologous proteins such as nitrogenase and the BchX protein of chlorophyllide oxidoreductase (Supplemental Fig. 2). The position and interactions of the N-terminal residues in our nucleotide-free structure suggests a possible auto-inhibitory role by forming a barrier to docking and shielding the [4Fe-4S] cluster of BchL.

\section{Amino acid substitutions in the flexible $N$-terminal region of BchL increases the rate of sub- strate reduction}

To test whether the flexible N-terminal region of BchL plays an auto-inhibitory role, based on the contacts observed in the crystal structure (Fig. 2d) and sequence alignments (Fig. 2e), we generated a singly mutated construct $\left(\mathrm{BchL}^{\mathrm{S} 17 \mathrm{~A}}\right)$ where Ser17 was mutated to Ala, and a quadruple mutated construct $\left(\mathrm{BchL}^{4 \mathrm{~A}}\right)$ where Asp13, Glu15, Ser17 and Gln19 were all substituted with Ala. Though density for residues 1-15 is not seen in any BchL structure, Ser17 and Gln19 are observed in our structure positioned across the $[4 \mathrm{Fe}-4 \mathrm{~S}]$ cluster (Supplemental Fig. 1b). Clarified cell lysates containing BchL ${ }^{\mathrm{S} 17 \mathrm{~A}}$ was mixed with Pchlide and BchNB and found to possess reductase activity after purification (Fig. 3b). However, amino acids substitutions in this N-terminal region affected 
protein stability: Both $\mathrm{BchL}^{\mathrm{S} 17 \mathrm{~A}}$ and $\mathrm{BchL}^{4 \mathrm{~A}}$ are soluble to a lesser degree compared to the wildtype protein and formed cloudy precipitates within 30 minutes of initiating reduction reactions. Substrate reducing activity with purified protein was assayed by mixing purified $\mathrm{BchL}, \mathrm{BchL}^{\mathrm{S} 17 \mathrm{~A}}$, or $\mathrm{BchL}^{4 \mathrm{~A}}(4 \mu \mathrm{M})$ with BchNB $(1 \mu \mathrm{M})$ and Pchlide $(35 \mu \mathrm{M})$ and spectroscopically monitoring the reaction over time in the absence or presence of ATP (3 mM). Pchlide and Chlide have characteristic absorbance maxima at $630 \mathrm{~nm}$ and $680 \mathrm{~nm}$, respectively, in aqueous solution. Formation of Chlide was observed as an increase in absorbance at $680 \mathrm{~nm}$ in the presence of ATP (Fig. 3b). Both $\mathrm{BchL}^{\mathrm{S} 17 \mathrm{~A}}$ and $\mathrm{BchL}{ }^{4 \mathrm{~A}}$ are active for substrate reduction and show Chlide formation rates $\sim 2$ 2.5 fold that of wild type $\mathrm{BchL}\left(\mathrm{k}_{\mathrm{obs}}=0.044 \pm 0.014,0.084 \pm 0.019\right.$, and $0.087 \pm 0.016 \mu \mathrm{M} / \mathrm{min}$ for $\mathrm{BchL}, \mathrm{BchL}{ }^{\mathrm{S} 17 \mathrm{~A}}$ and $\mathrm{BchL}{ }^{4 \mathrm{~A}}$, respectively; Fig. 3c). We speculate that actual differences in activity are likely much larger as the effective concentrations of the mutant BchL proteins are likely lower than calculated during the experiment due to protein instability. We also generated a truncated version of BchL missing the first 27 amino acids $\left(\mathrm{BchL}^{\mathrm{N} \Delta 27}\right)$. We were successfully able to overexpress $\mathrm{BchL}{ }^{\mathrm{N} \Delta 27}$, however it was poorly soluble, and we were unable to obtain sufficiently pure protein for biochemical studies. These difficulties provide additional evidence that the flexible N-terminus might play an important role in BchL function and stability. We also recently showed that the presence of a functional Suf operon in E. coli cells leads to a 3-fold enhancement of BchL overproduction suggesting that the flexible N-terminus might be modulated by specific cellular mechanisms. ${ }^{12}$

\section{ATP-binding causes changes in the local environment of the BchL [4Fe-4S] cluster affecting their EPR spectral line-shape and intensities}


Since the flexible N-terminal region binds to the BchNB interaction interface within BchL, we hypothesized that ATP binding could promote conformational changes in BchL to relieve autoinhibition. We thus used electron paramagnetic resonance (EPR) to probe local changes in the [4Fe-4S] cluster environment, which includes the binding site for the $\mathrm{N}$-terminus. Interpretation of the g-tensors of $[4 \mathrm{Fe}-4 \mathrm{~S}]$ clusters in direct structural terms is rarely possible, ${ }^{13}$ but EPR can nevertheless provide information about relative conformational changes around the cluster. Previous studies have described the $[4 \mathrm{Fe}-4 \mathrm{~S}]$ cluster of BchL as an axial species, ${ }^{5,6,14}$ while others reported a rhombic species. ${ }^{15,16}$ Here, EPR at different temperatures indicated that two distinct EPR signals were exhibited by the [4Fe-4S] cluster (Supplemental Fig. 3a). At $5 \mathrm{~K}$, a signal termed $\mathrm{FeS}^{\mathrm{A}}$ was observed that appears axial but was best simulated with rhombic $g$-values of $2.00,1.94$, and 1.85; both $g_{1}$ and $g_{3}$ are atypically low for a prototypical Cys4-ligated [4Fe-4S] cluster, and the associated resonances exhibit large line widths (Supplemental Fig. 3a). This signal was very fast-relaxing and was no longer detectable at $17.5 \mathrm{~K}$. At this higher temperature $(17.5 \mathrm{~K})$, a more typical rhombic signal, $\mathrm{FeS}^{\mathrm{B}}$, with $g_{1,2,3}=2.04,1.94$, and 1.89 was observed, and at an intermediate temperature $(10 \mathrm{~K})$ the observed signal was well replicated by a 40\%:60\% mixture of the simulations of $\mathrm{FeS}^{\mathrm{A}}$ and $\mathrm{FeS}^{\mathrm{B}}$, respectively. As expected for a [4Fe-4S] cluster, the EPR signals were fast-relaxing and were not detectable at $30 \mathrm{~K}$ or higher temperature. BchL exhibited analogous signals to $\mathrm{FeS}^{\mathrm{A}}$ and $\mathrm{FeS}^{\mathrm{B}}$ when incubated with BchNB and ADP (Supplemental Figs. 3b and 3c) although, notably, both $\mathrm{ADP}$ and $\mathrm{BchNB}$ served to increase the relaxation rate of the $\mathrm{FeS}^{\mathrm{B}} \mathrm{EPR}$ signal, suggesting a more efficient coupling of the cluster to the lattice via increased strain energy. In addition, with $\mathrm{ADP}$, the proportion of the $\mathrm{FeS}^{\mathrm{A}}$ species was diminished by a factor of two. Based on the structural data for $\mathrm{BchL}$ and the relaxation properties of the EPR signals, we propose the $\mathrm{FeS}^{\mathrm{A}}$ species as having a 'cap' across the cluster, formed by the flexible N-terminus, whereas the 
$\mathrm{FeS}^{\mathrm{B}}$ species is uncapped; in solution, these two species are likely in dynamic equilibrium. Upon the addition of ATP, the relaxation rate of the $\mathrm{FeS}^{\mathrm{B}}$ species was further enhanced and was undetectable at $17.5 \mathrm{~K}$ (Supplemental Fig. 3c), while the $\mathrm{FeS}^{\mathrm{A}}$ signal exhibited rapid-passage distortion at $5 \mathrm{~K}$, indicating a diminution of the relaxation rate for that species. These data suggest that ATP binding increases the conformational strain of uncapped $\mathrm{FeS}^{\mathrm{B}}$ and somewhat inhibits the strong interaction of the cap with the cluster in $\mathrm{FeS}^{\mathrm{A}}$. The $\mathrm{FeS}^{\mathrm{A}} \mathrm{EPR}$ signals from the $\mathrm{BchL}^{\mathrm{S} 17 \mathrm{~A}}$ variant (Supplemental Fig. 3C) exhibited strong rapid-passage distortion at $5 \mathrm{~K}$ and overall reduced signal intensities over the 5 - $17.5 \mathrm{~K}$ temperature range; the addition of ATP restored the intensity of the $\mathrm{FeS}^{\mathrm{B}}$ signal somewhat, suggesting that relaxation properties were responsible for this phenomenon and that, therefore, the interaction of the cap region with the cluster is altered in $\mathrm{BchL}^{\mathrm{S} 17 \mathrm{~A}}$. The conformational changes around the $[4 \mathrm{Fe}-4 \mathrm{~S}]$ cluster in BchL upon ATP binding appear similar to the $\mathrm{BchL}^{\mathrm{S} 17 \mathrm{~A}}$ protein in the absence of ATP (Fig. 3c). Based on these results, we propose that the loss of auto-inhibition drives higher overall substrate reduction activity in $\mathrm{BchL}^{\mathrm{S} 17 \mathrm{~A}}$.

\section{A DFD amino-acid patch promotes inter-subunit cross-stabilization upon ATP binding}

The ATP-binding sites in BchL are situated away from the BchL:BchNB interaction interface (Fig. 1a, Fig. 2a-c). Thus, we hypothesized that other regions between these two sites might function as a conduit for communication and be an important contributor to potential conformational changes and promote rearrangement of the flexible N-terminus. Comparison of the nucleotide-free (Fig. 4a), ADP-bound (Fig. 4b) ${ }^{8}$ and ADP-AlF3-NB-bound (Fig. 4c) ${ }^{9}$ BchL crystal structures reveal a 'DFD patch' composed of amino acid residues Asp180, Phe181, Asp182 that undergo rearrangements and contact the hydroxyl groups of the sugar moiety of the bound nucleotide. Interestingly, the contacts only appear in the ADP-AlF $3-\mathrm{L}-\mathrm{NB}$ DPOR structure (Fig. 4c), and thus 
the DFD patch appears poised to be important for coordinating ATP-dependent conformational changes during complex formation and substrate reduction. D180 and D182 from one subunit form a network of interactions in trans with the sugar moiety of ATP bound to the neighboring subunit along with R244 in the nucleotide-bound subunit (Fig. 4c). We have termed this series of interactions "inter-subunit cross stabilization of ATP". These interactions appear to be stabilized in the transition state when BchL forms a complex with BchNB. One possibility is that ATP-binding drives inter-subunit cross stabilization and would provide the necessary conformational stability required to pry away the flexible N-terminal tail from binding across the $[4 \mathrm{Fe}-4 \mathrm{~S}]$ cluster, thus relieving inhibition. If this were the case, mutations in the DFD patch would not affect ATP binding, but would perturb substrate reduction.

To test this hypothesis, and the role of the DFD patch, we generated three BchL variants carrying amino acid substitutions wherein Asp180 (BchL $\left.{ }^{\mathrm{D} 180 \mathrm{~N}}\right)$, Asp182 (BchL $\left.{ }^{\mathrm{D} 182 \mathrm{~N}}\right)$, or both, $\left(\right.$ BchL $\left.^{\text {DFD-NFN }}\right)$ were substituted to Asn. Phe181 does not make contacts with the sugar and thus was not perturbed. BchL ${ }^{\mathrm{D} 180 \mathrm{~N}}$ was poorly active for Pchlide reduction (Fig. 4d, e) whereas $\mathrm{BchL}^{\mathrm{D} 182 \mathrm{~N}}$ and BchL ${ }^{\text {DFD-NFN }}$ were inactive (Fig. 4d, e), suggesting that both residues are important for function. Next, to precisely understand why mutations in the DFD patch affected substrate reduction, we analyzed the nucleotide binding, and EPR spectral properties of BchL ${ }^{\text {DFD-NFN }}$. Nucleotide binding was measured by capturing the BchL:ATP complex using radiolabeled $\alpha^{32} \mathrm{P}-\mathrm{ATP}$ in a nitrocellulose filter binding assay. BchL binds to the nitrocellulose filter and ATP bound to the protein is retained on the membrane, while unbound ATP flows through the filter. Both BchL and $\mathrm{BchL} \mathrm{DFD}^{\mathrm{DFN}}$ are capable of binding to ATP, and no ATP is retained on the membrane when no protein is present in the reaction (Fig. 4f). This finding is consistent with the ADP-bound crystal structure where no contacts between the DFD patch and nucleotide are observed (Fig. 4b). Thus, 
the loss in substrate reduction activity in the BchL ${ }^{\text {DFD-NFN }}$ protein occurs post ATP binding, and possibly due to a loss of the promotion of conformational changes necessary for multiple rounds of complex formation with BchNB.

EPR of BchL ${ }^{\text {DFD-NFN }}$ at $5 \mathrm{~K}$ (Supplemental Figure 3e and Fig. 4g) again revealed an FeS signal, though the rapid-passage distortions indicated that ATP binding enhanced relaxation in BchL ${ }^{\text {DFD-NFN }}$ whereas ATP inhibits relaxation in native BchL. The overall relaxation rates for FeS ${ }^{\mathrm{A}}$ in native $\mathrm{BchL}$ and $\mathrm{BchL}{ }^{\text {DFD-NFN }}$ can be summarized as $\mathrm{BchL}>\mathrm{BchL}-\mathrm{ATP} \approx \mathrm{BchL}{ }^{\text {DFD-NFN }}-\mathrm{ATP}>$ BchL ${ }^{\text {DFD-NFN }}$. At $10 \mathrm{~K}$, the EPR spectra of both native BchL and BchL ${ }^{\text {DFD-NFN }}$ are almost indistinguishable and consist of $60 \% \mathrm{FeS}^{\mathrm{B}}$, whereas the ATP complexes of both exhibit EPR signals indistinguishable from each other but containing only $35 \% \mathrm{FeS}^{\mathrm{B}}$ and $65 \%$ of the relaxation-inhibited $\mathrm{FeS}^{\mathrm{A}}$ (Supplemental Figure 5e). The spectra of both native BchL and BchL ${ }^{\text {DFD-NFN }}$ at $17.5 \mathrm{~K}$ are again indistinguishable and are due to $\mathrm{FeS}^{\mathrm{B}}$ alone (Supplemental Figure 5e). In both cases, the signals are of diminished intensity upon addition of ATP due to enhanced relaxation, to about $25 \%$ in the case of BchL ${ }^{\text {DFD-NFN }}$-ATP and almost completely extinguished in native BchL-ATP. It is not clear whether the residual $\mathrm{FeS}^{\mathrm{B}}$ signal from BchL ${ }^{\mathrm{DFD}-\mathrm{NFN}}-\mathrm{ATP}$ at $17.5 \mathrm{~K}$ is due to slower relaxation than in native BchL-ATP or less than stoichiometric binding of ATP. So, overall, relaxation of $\mathrm{FeS}^{\mathrm{A}}$ is markedly inhibited in $\mathrm{BchL}{ }^{\mathrm{DFD}-\mathrm{NFN}}$, indicating poorer coupling to a strained lattice in the capped species, while the cluster environments in the uncapped $\mathrm{FeS}^{\mathrm{B}}$ species of native BchL and BchL ${ }^{\text {DFD-NFN }}$ are indistinguishable by EPR (Fig. 4g); and the binding of ATP to both species provides very similar cluster environments for both $\mathrm{FeS}^{\mathrm{A}}$ and $\mathrm{FeS}^{\mathrm{B}}$. Thus, ATP binding to BchL ${ }^{\text {DFD- }}$ NFN does not elicit the complete portfolio of conformational changes required for substrate reduction. 


\section{Binding of ATP to both subunits in the BchL dimer are required to generate a concerted motion}

\section{to promote inter-subunit cross stabilization and drive substrate reduction}

Each BchL homodimer consists of two sites for ATP binding (Fig. 5a), and the two subunits are covalently tethered by a single [4Fe-4S] cluster (Fig. 2a-c). In the homologous Fe protein of nitrogenase, the sites have been shown to bind to nucleotide with differing affinities. ${ }^{17}$ A crystal structure of the Fe-protein with two different nucleotides occupying the dimer has also been solved suggesting that the two ATP sites could play distinct roles in substrate reduction ${ }^{18}$ (Supplemental Fig. 3c). To test the functional role of the two ATP-binding sites in BchL, we generated a covalently linked version of BchL by expressing the two subunits as a single polypeptide. The "linked" construct included the same N-terminal 6x-poly histidine tag followed by a TEV protease site identical to all other L-protein constructs. Two BchL subunits were tethered by a flexible linker that connected the $\mathrm{C}$-terminal end of the first monomer to the N-terminal end of the other (Fig. 5b). Covalent linkage of multimers can interfere with activity due to a variety of effects including conformational strain, non-specific interactions due to the linker, introduction of non-native secondary structures, or undefined effects. The linker was therefore constructed to carry TEV protease recognition sites bookending the linker region enabling proteolytic removal if the intact linker interfered with activity for any reason. (Fig. 5b). We found that the length of the linker is a key determinant of protein activity. Linkers shorter than 15 amino acids are defective for substrate reduction, and optimal activity is obtained when linker lengths are longer than 20 amino acids (Supplemental Fig. 4c, d). The optimized linked-BchL behaved similarly to wild-type BchL during

purification (Fig. 5c) and was stable and fully active for Pchlide reduction (Fig. 5d). Removal of the linker after protease cleavage also resulted in protein activity similar to the uncleaved and wildtype BchL proteins (Supplemental Fig. 4b). These results show that a linker of optimal length does 
not interfere with protein function. The linked L-protein appeared to have slightly faster activity. We believe that this is due to only one N-terminal tail being free to "cap" the [4Fe-4S] cluster, whilst the other is attached to the C-terminus of the first monomer and likely does not contribute to auto-inhibition of activity. This suggests that the 2 available $\mathrm{N}$-terminal tails in the unlinked construct are dynamically interacting with the cluster.

Next, to assess the contribution of the individual ATP sites, we generated an ATP-binding deficient mutation in one or both subunits in linked-BchL (Fig. 5e). Substitution of the conserved Lys44 with an Ala in the Walker A motif perturbs ATP binding (Fig. 5a). Using filter binding analysis, we determined the amount of ATP bound to the linked and unlinked versions of BchL. BchL (L) and linked-BchL with unaltered ATP binding sites $\left(\mathrm{L}_{1} \mathrm{~L}_{2}\right)$ bind ATP to similar extents (Fig. 5f). The K44A substitution in the Walker-A ATP binding pocket of BchL $\left(\mathrm{L}^{\mathrm{KA}}\right)$ and in both subunits of the linked-BchL $\left(\mathrm{L}_{1}{ }^{\mathrm{KA}}-\mathrm{L}_{2}{ }^{\mathrm{KA}}\right)$ abolishes ATP binding as expected (Fig. 5f). When only one of the two ATP binding sites are mutated $\left(\mathrm{L}_{1}{ }^{\mathrm{KA}}-\mathrm{L}_{2}\right)$, partial ATP binding is observed (Fig. 5f). When ATP binding is perturbed in both sites or in just one site, a complete loss of Pchlide reduction activity is observed (Fig. 5g, h). These data suggest that both ATP molecules in the BchL dimer are required for substrate reduction. Based on these data, we propose that binding of both ATP molecules likely causes cooperative conformational changes in the two halves of the L-protein homodimer and drive inter-subunit cross stabilization.

\section{Discussion}


Nitrogenase and nitrogenase-like enzymes such as DPOR and Chlorophyllide Oxidoreductase (COR) share structural similarity with respect to their electron donor and electron acceptor component proteins. These proteins catalyze multiple rounds of ET for substrate reduction, and transient association of the electron donor and electron acceptor is a prerequisite for each ET event. ATP binding to the electron donor is canonically assigned as the mechanistic trigger that promotes the assembly of the component proteins. However, the precise structural and functional principles underlying this ATP-driven process have largely remained unclear. The results presented in this study shed light on several ATP-binding driven changes in BchL that enable DPOR to function in an oxygenic environment.

In the nucleotide-free crystal structure, residues 16-29 are ordered in one of the four chains and suggest a novel regulatory role for the flexible N-terminus of BchL. Interestingly, the N-terminal tail binds across the $[4 \mathrm{Fe}-4 \mathrm{~S}]$ cluster and appears poised to block the docking surface for interaction with BchNB (Fig. 2a-c). Six residues of each BchL chain are thought to interact with BchNB to form the DPOR complex. In our BchL structure, the disordered N-terminus from one subunit forms an inhibitory barrier across the docking surface, and hydrogen bonding affects three of the 12 docking residues (Cys 126, Gly 161, and Gln 168), suggesting that the N-terminus forms a barrier to docking and ET to BchNB. Mutations that perturb specific interactions in this region enhance the substrate reduction activity, supporting an auto-inhibitory role for this region in DPOR function (Fig. 3). Although binding of both ADP and ATP can be thought to trigger conformational changes leading to the displacement of N-terminal residues, only ATP hydrolysis drives the dissociation of the BchL-NB complex to complete the catalytic cycle. Therefore, the conformational change in the $\mathrm{N}$-terminal region could be yet another way of coupling ATP-binding to BchL with Pchlide reduction in $\mathrm{BchNB}$. 
A BLAST-P analysis of residues 1-29 yielded BchL protein hits spread across $~ 150$ species of bacteria. A nine-residue patch in the flexible N-terminus [DGEGSVQVH] (residues 13-21 in $R$. capsulatus BchL) is highly conserved, supporting functional significance (Fig. 2e). The flexible $\mathrm{N}$-terminal region is unique to the DPOR system. The tail is not conserved in COR which catalyzes the subsequent reductive step in chlorophyll synthesis (Fig. 2e and Supplemental Fig. 2), and the entire N-terminal region does not exist in the Fe-protein of nitrogenase. The biological necessity for such a regulatory region remains to be established, though may exist as a mechanism to prevent off-target electron donation and the formation of free radicals in the cell. The observations that substitutions in this region affect BchL stability in vitro suggests a potential role in protecting the [4Fe-4S] cluster against oxidation and off-target electron donation.

ATP binding to both subunits of BchL promotes a network of interactions between a conserved DFD patch and the sugar moiety of the nucleotide. This generates an inter-subunit cross stabilization as the DFD patch from one subunit contacts the nucleotide bound to the other. Such an ATP-binding dependent conformational change could generate an upward compaction of BchL leading to the release of the auto-inhibitory N-terminus from the docking interface and promote complex formation with BchNB (Fig. 6). Substitutions in either the DFD patch or rendering one subunit devoid for ATP binding shuts down substrate reduction activity. We propose a model where cooperative interactions between the DFD patch and the nucleotide relieves the auto-inhibition by the $\mathrm{N}$-terminus of $\mathrm{BchL}$ and is a key regulatory step in transient assembly of the DPOR complex (Fig. 6). In the related nitrogenase complex, several crystal structures of the homologous Fe-protein have been solved in complex with a variety of nucleotides in the absence and presence of the MoFe-protein. In these structures, the relative distances between the two nucleotides does not change (Supplemental Fig. 3). Since the flexible N-terminal region is not conserved in the Fe- 
protein of, it may indicate the presence of a different protective mechanism in the nitrogenase system.

In DPOR, the subtle structural changes observed between the ADP-bound and the nucleotide-free crystal structures indicate how nucleotide-binding enables BchL to carry out ET to BchNB. Predictable changes occur around the ATP-binding pocket: Comparison of the two structures suggest that Switch II region may act as a redox switch. The Switch II region is fully conserved between BchL and NifH (the nitrogenase Fe protein), including Phe135 in NifH (Phe163 in $\mathrm{BchL}$ ), which has been demonstrated to be important for the redox properties of the [4Fe-4S] cluster of NifH. ${ }^{19}$ In the absence of ADP, the local environment immediately surrounding the BchL-cluster is more packed and hydrophobic, promoted by the interactions of Leu155, Val158, and Phe163 (Supplemental Fig. 6). In general, for redox-active metal centers, hydrophobicity increases reduction potential, ${ }^{20,21}$ suggesting that in the absence of $\mathrm{Mg}$-ADP, the BchL-cluster is less likely to become oxidized. While electrochemical data is not available for BchL, studies with NifH show that in the presence of ATP or ADP, the midpoint reduction potential is $120-160 \mathrm{mV}$ more negative,${ }^{19}$ indicating that oxidation of the $[4 \mathrm{Fe}-4 \mathrm{~S}]$ cluster of $\mathrm{NifH}$ for electron transfer to the $\mathrm{P}$ cluster becomes more favorable in the presence of nucleotides. The conservation of Switch II sequence suggests that the redox properties of the $[4 \mathrm{Fe}-4 \mathrm{~S}]$ cluster of BchL could be similarly modulated. The van der Waals interaction observed between the [4Fe-4S] cluster of BchL, its ligand Cys160, and Phe163 (Fig. 2c) could be a means of modulating the redox properties of the BchL-[4Fe-4S] cluster in the presence and absence of nucleotides.

Photochemistry and chlorophyll biogenesis occur in the presence of dissolved molecular $\mathrm{O}_{2}$ that can generate reactive oxygen species (ROS) such as singlet oxygen and peroxide species. In fact, an increase in BchL transcription occurs when cells encounter singlet oxygen. ${ }^{22}$ The DPOR 
system thus must maintain functionality in oxygenic environments despite the ability of reactive oxygen species to destroy the [4Fe-4S] cluster necessary for electron transfer. The selective evolution of the flexible $\mathrm{N}$-terminal region and the protective role it plays by binding across the [4Fe4 S ] cluster are likely functional adaptations to enable DPOR to function in oxygenic environments. In the case of DPOR, ATP binding relieves autoinhibition. Intriguingly, such protective mechanisms are not found in the Fe-protein of nitrogenase as nitrogen reduction predominantly functions under anaerobic conditions. It is interesting to note that the N-terminal region is not strictly conserved in BchX of the COR enzyme complex, which catalyzes the subsequent reduction of Chlide. Whether the $\mathrm{N}$-terminus of BchX plays a similar role in capping the $[4 \mathrm{Fe}-4 \mathrm{~S}]$ cluster remains to be established.

\section{Materials and Methods}

Reagents and Buffers. Chemicals were purchased from Sigma-Millipore Inc. (St. Louis, MO), Research Products International Inc. (Mount Prospect, IL) and Gold Biotechnology Inc. (St. Louis, MO). Oligonucleotides for cloning were purchased from Integrated DNA Technologies (Coralville, IA). Enzymes for molecular biology were purchased from New England Biolabs (Ipswich, MA). All reagents and buffers were thoroughly degassed using alternating cycles of vacuum and nitrogen pressure on a home built Schlenk line apparatus. Anaerobic conditions were maintained via airtight syringes, excess reductant and a vinyl glove box (Coy Laboratories, MI) under a Nitrogen $(95 \%)$ Hydrogen (5\%) mix atmosphere. 
Generation of protein overexpression constructs. The coding regions for $\mathrm{BchL}, \mathrm{BchN}$, and $\mathrm{BchB}$ were PCR amplified from Rhodobacter sphaeroides genomic DNA and cloned into pRSF-Duet 1 or pET-Duet 1 plasmids as described. ${ }^{12}$ Mutations in BchL were generated using Q5 site-directed mutagenesis (New England Biolabs, Ipswich, MA). Plasmids used to express the linked-BchLproteins carrying glycine linkers of various lengths were synthesized as codon-optimized genes (Genscript Inc., Piscataway, NJ). The longest iteration of the linked-BchL-protein was generated as described in the supplemental section.

Protein purification. BchL and BchNB proteins were overexpressed and purified as originally described, ${ }^{23}$ with modifications as recently reported. ${ }^{12}$ The following additional steps were added to the purification of the linked-L-proteins. During cell lysis and all subsequent steps, protease inhibitors (protease inhibitor cocktail, Millipore-Sigma Inc - catalog \#P2714) and 1 mM PMSF were added to all buffers. As an additional purification step, the concentrated linked-L-protein from the Q-Sepharose eluate was subsequently fractionated over a Sephadex S200 26/600 PG (GE Life Sciences) column using STD buffer (100 mM HEPES, pH 7.5, $150 \mathrm{mM} \mathrm{NaCl,} 10 \mathrm{mM} \mathrm{MgCl} 2,1.7$ $\mathrm{mM}$ sodium dithionite, $1 \mathrm{mM}$ PMSF and protease inhibitors). Protein concentrations were determined using the Bradford assay and Bovine Serum Albumin as a standard.

Generation of Pchlide. Pchlide was generated from a Rhodobacter sphaeroides ZY-5 strain harboring a deletion of the BchL gene (a kind gift from Dr. Carl Bauer, Indiana University) ${ }^{24}$ and purified as described. ${ }^{12}$ 
Pchlide Reduction Assays. Reduction of Pchlide to Chlide was measured spectroscopically by mixing BchNB (5 $\mu \mathrm{M}$ tetramer), BchL (20 $\mu \mathrm{M}$ dimer), and $35 \mu \mathrm{M}$ Pchlide, in the absence or presence of ATP (3 mM) in STD buffer with $10 \mathrm{mM} \mathrm{MgCl}_{2}$. Substrate reduction experiments were carried out in $200 \mu \mathrm{l}$ reactions and quenched at the denoted timepoints with $800 \mu \mathrm{l}$ of $100 \%$ acetone. The acetone/reaction mixture was spun down in a table-top centrifuge at 13,226 x $\mathrm{g}$ for 4 minutes. The supernatant was transferred to a cuvette and absorbance scans from $600 \mathrm{~nm}$ to $725 \mathrm{~nm}$ were recorded on a Cary $100 \mathrm{UV}-$ Vis spectrophotometer (Agilent Technologies, Santa Clara, CA). Chlide appearance was quantified using the molar extinction coefficient 74,900 $\mathrm{M}^{-1} \mathrm{~cm}^{-1}$ at 666 nm. For substrate reduction experiments shown in Figure 3 Chlide appearance was measured in aqueous solution inside a Type 41 macro cuvette with a screw cap (Firefly Scientific, Staten Island, NY). Reactions contained BchNB (1 $\mu \mathrm{M}$ tetramer), BchL (4 $\mu \mathrm{M}$ dimer), $35 \mu \mathrm{M}$ Pchlide, and ATP (3 mM) in STD buffer containing $10 \mathrm{mM} \mathrm{MgCl}_{2}$. Reactions were initiated by addition of degassed ATP via a gas-tight syringe, and spectra were recorded from 400-800nm every 60 s as described above. Figure 3B shows absorbance values from difference spectra generated by subtracting timepoints from the first spectra recorded before ATP addition.

BchL crystallization. BchL crystals were grown anaerobically $\left(100 \% \mathrm{~N}_{2}\right.$ environment with $<0.1$ ppm $\mathrm{O}_{2}$ ) inside a Unilab Pro glovebox (mBraun, Stratham, $\mathrm{NH}$ ) at $15{ }^{\circ} \mathrm{C}$ using the vapor diffusion method. ${ }^{25}$ All materials and buffers were pre-treated to remove oxygen as previously described. ${ }^{25}$ Initial sparse matrix screens were set up anaerobically using a Mosquito Crystal robotic liquid handler (TTP Labtech, Boston, MA). $1 \mu \mathrm{L}$ of $200 \mathrm{mM}$ sodium dithionite solution was added to every well to ensure complete removal of any dissolved oxygen. For each drop, $200 \mathrm{~nL}$ of well solution and $200 \mathrm{~nL}$ of $100 \mu \mathrm{M}$ dimeric BchL (in $100 \mathrm{mM}$ HEPES pH 7.5, $150 \mathrm{mM} \mathrm{NaCl,} \mathrm{10 \%}$ (v/v) glycerol) were mixed. A crystal was observed after approximately one month with the well 
solution consisting of 0.6 M sodium chloride, 0.1 M MES:NaOH pH 6.5, $20 \%$ (w/v) PEG 4000.

Larger volume (3-4 $\mu \mathrm{L})$ drops of the same well solution and protein concentration in 1:1, 1:2 and

$2: 1$ ratios of protein to well solution yielded large single crystals after $\sim 2-3$ months. Prior to freezing, the well solution was mixed in an equal volume of cryoprotectant solution with a final concentration of $9 \%$ sucrose (w/v), $2 \%$ glucose (w/v), $8 \%$ glycerol (v/v), and $8 \%$ ethylene glycol (v/v). Crystals were soaked for a few seconds in the cryoprotectant before being cryo-cooled in liquid nitrogen. A crystal from a drop set up with $2 \mu \mathrm{L}$ well solution and $2 \mu \mathrm{L}$ protein solution was used for in-house data collection, while different crystals that grew with $2 \mu \mathrm{L}$ well solution and $1 \mu \mathrm{L}$ protein solution were used for synchrotron data collection.

BchL data collection, processing and refinement. An initial model was built using data collected with an in-house Rigaku MicroMax 007HF X-ray source equipped with a Pilatus 300K detector. A complete dataset at cryogenic temperature $(100 \mathrm{~K})$ was collected to $2.92-\AA$ resolution, which was integrated using HKL2000 26 and merged and scaled using SCALA in the CCP4 suite. ${ }^{27}$ Phase determination was initially estimated through molecular replacement (PHASER) using the Rhodobacter sphaeroides ADP-bound BchL structure (PDB ID: 3FWY, with all ligands removed) as the search model. ${ }^{8,28}$ A solution was found with two dimers in the asymmetric unit. Following molecular replacement, rigid-body refinement was performed in Phenix. ${ }^{28}$ A starting model was built using AutoBuild and further improved with iterative rounds of model building and refinement using $\mathrm{COOT}^{29}$ and Phenix.

Higher resolution data were collected on a different crystal at beamline 17-ID-1 (AMX), National Synchrotron Lightsource-II, at the Brookhaven National Laboratory on a Dectris Eiger 9M detector. Two complete datasets collected at 100K were integrated, scaled and merged to 2.6- 
A resolution using HKL2000. The partially refined model from home-source data was used as a molecular replacement model for solving the structure in Phenix. The resulting model was improved through iterative rounds of model building using COOT and Phenix. Data processing and refinement statistics are presented in Supplemental Table 1.

ATP binding assay. Nitrocellulose membranes, cut into $2 \times 2 \mathrm{~cm}$ squares, were pretreated with $0.5 \mathrm{~N} \mathrm{NaOH}$ for $2 \mathrm{~min}$, washed extensively with $\mathrm{H}_{2} \mathrm{O}$, and equilibrated in binding buffer (100 mM Hepes, $\mathrm{pH} 7.5,150 \mathrm{mM} \mathrm{NaCl}$, and $\left.10 \mathrm{mM} \mathrm{MgCl}_{2}\right)$. In the reactions $(100 \mu \mathrm{L}), \mathrm{BchL}(4 \mu \mathrm{M})$ was incubated with $1 \mathrm{mM} \mathrm{ATP}+0.3 \mu \mathrm{Ci} \alpha^{32} \mathrm{P}$-ATP for $10 \mathrm{~min}$ at $25^{\circ} \mathrm{C}$, and $20 \mu \mathrm{L}$ aliquots of the reaction were filtered through the membrane on a single filter holder (VWR Scientific Products). The membranes were washed before and after filtration with $250 \mu \mathrm{L}$ of nucleotide binding buffer and air dried before overnight exposure onto a PhoshorImaging screen. $1 \mu \mathrm{L}$ aliquots were spotted onto a separate membrane to measure total nucleotide in the reaction. Radioactivity on the membrane was quantitated on a PhosphorImager (GE Life Sciences). Total ${ }^{32} \mathrm{P}-\mathrm{ATP}$ bound was calculated using the following equation: [[bound $\left.{ }^{\text {signal }}\right] /\left[\left[\right.\right.$ total $\left.\left.\left.\left.^{\text {signal }}\right] \times 20\right]\right]\right] \times[$ ATP $]$

EPR Spectroscopy. EPR spectra were obtained at 5,10, 17.5, and $30 \mathrm{~K}$ on an updated Bruker EMXAA-TDU/L spectrometer equipped with an ER4112-SHQ resonator $(9.48 \mathrm{GHz})$ and an HP 5350B microwave counter for precise frequency measurement. Temperature was maintained with a ColdEdge/Bruker Stinger S5-L recirculating helium refrigerator, and an Oxford ESR900 cryostat and MercuryITC temperature controller. Spectra were recorded with either $0.3 \mathrm{G}\left(3 \times 10^{-5} \mathrm{~T}\right)$ or $1.2 \mathrm{G}$ $(0.12 \mathrm{mT})$ digital field resolution with equal values for the conversion time and the time constant, $1.0 \mathrm{~mW}$ incident microwave power, and $12 \mathrm{G}(1.2 \mathrm{mT})$ magnetic field modulation at $100 \mathrm{kHz}$. EPR simulations were carried out using Easyspin. ${ }^{30}$ 
Samples for EPR Spectroscopy. $200 \mu \mathrm{l}$ EPR samples contained $40 \mu \mathrm{M}$ BchL (or mutant), 1.7mM dithionite, and, where indicated 3mM ATP, 3mM ADP and/or 20uM BchNB. For a subset of the experiments, EPR experiments were carried out with $20 \mu \mathrm{M}$ BchNB, $40 \mu \mathrm{M}$ Pchlide, and, where indicated, $3 \mathrm{mM}$ ATP. Protein samples were prepared and transferred to the EPR tubes in the glove box and stoppered with a butyl rubber stopper. Samples were removed from the glove box and immediately flash frozen in liquid nitrogen and then analyzed by EPR.

\section{Acknowledgements}

The authors thank Gabrielle Illava for performing supporting experiments and Phil Jeffrey (Princeton Macromolecular Diffraction Facility) for assistance with crystallographic data collection. Additionally, the authors would like to thank Amanda Byer for her critical reading of the manuscript.

\section{Author Contributions}

EIC, SMSI, MBW, SO, RK, KD, BB and MS performed experiments. EIC, SMSI, MBW, JPB, LCS, NA, BB and EA designed experiments, and performed data analysis. EA, NA, BB, and EIC primarily wrote the manuscript.

\section{Funding}

This work was supported by a grant from the Department of Energy, Office of Science, Basic Energy Sciences (DE-SC0017866) to E.A. and the Biological Electron Transfer and Catalysis (BETCy) Energy Frontiers Research Center (EFRC-DE-SC0012518) to L.C.S. This research used 
beamline 17-ID-1 at the National Synchrotron Light Source II (NSLS-II), a U.S. Department of Energy (DOE) Office of Science User Facility operated for the DOE Office of Science by Brookhaven National Laboratory under Contract No. DE-SC0012704. The Life Science Biomedical Technology Research resource at NSLS-II is primarily supported by the National Institute of Health, National Institute of General Medical Sciences (NIGMS) through a Biomedical Technology Research Resource P41 grant (P41GM111244), and by the DOE Office of Biological and Environmental Research (KP1605010). This work was also supported by National Institutes of Health grant GM124847 (to N.A.) and startup funds from Princeton University and Cornell University (to N.A.). EPR was supported by an NSF Major Research Instrumentation award (CHE1532168) to B.B. and by Bruker Biospin. E.I.C was supported by a GANN fellowship from the department of education and the Arthur J. Schmitt fellowship from Marquette University. 


\section{References}

1. Fujita, Y., Takagi, H. \& Hase, T. Cloning of the gene encoding a protochlorophyllide reductase: the physiological significance of the co-existence of light-dependent and independent protochlorophyllide reduction systems in the cyanobacterium Plectonema boryanum. Plant Cell Physiol 39, 177-85 (1998).

2. Fujita, Y., Takagi, H. \& Hase, T. Identification of the chlB gene and the gene product essential for the light-independent chlorophyll biosynthesis in the cyanobacterium Plectonema boryanum. Plant Cell Physiol 37, 313-23 (1996).

3. Reinbothe, C. et al. Chlorophyll biosynthesis: spotlight on protochlorophyllide reduction. Trends Plant Sci 15, 614-24 (2010).

4. Nomata, J. et al. Dark-operative protochlorophyllide oxidoreductase generates substrate radicals by an iron-sulphur cluster in bacteriochlorophyll biosynthesis. Sci Rep 4, 5455 (2014).

5. Brocker, M.J. et al. Crystal structure of the nitrogenase-like dark operative protochlorophyllide oxidoreductase catalytic complex (ChlN/ChlB)2. J Biol Chem 285, 27336-45 (2010).

6. Muraki, N. et al. X-ray crystal structure of the light-independent protochlorophyllide reductase. Nature 465, 110-4 (2010).

7. Nomata, J., Terauchi, K. \& Fujita, Y. Stoichiometry of ATP hydrolysis and chlorophyllide formation of dark-operative protochlorophyllide oxidoreductase from Rhodobacter capsulatus. Biochem Biophys Res Commun 470, 704-709 (2016). 
8. Sarma, R. et al. Crystal structure of the L protein of Rhodobacter sphaeroides lightindependent protochlorophyllide reductase with MgADP bound: a homologue of the nitrogenase Fe protein. Biochemistry 47, 13004-15 (2008).

9. Moser, J. et al. Structure of ADP-aluminium fluoride-stabilized protochlorophyllide oxidoreductase complex. Proc Natl Acad Sci U S A 110, 2094-8 (2013).

10. Duval, S. et al. Electron transfer precedes ATP hydrolysis during nitrogenase catalysis. Proc Natl Acad Sci U S A 110, 16414-9 (2013).

11. Krissinel, E. \& Henrick, K. Inference of macromolecular assemblies from crystalline state. J Mol Biol 372, 774-97 (2007).

12. Corless, E.I., Mettert, E.L., Kiley, P.J. \& Antony, E. Elevated Expression of a Functional Suf Pathway in E. coli BL21(DE3) Enhances Recombinant Production of an Iron-Sulfur Cluster Protein. Journal of Bacteriology, JB.00496-19 (2019).

13. Hagen, W.R. EPR Spectroscopy of Iron-Sulfur Proteins. Advances in Inorganic Chemistry 38, 165-222 (1992).

14. Brocker, M.J. et al. ATP-driven reduction by dark-operative protochlorophyllide oxidoreductase from Chlorobium tepidum mechanistically resembles nitrogenase catalysis. J Biol Chem 283, 10559-67 (2008).

15. Nomata, J., Kitashima, M., Inoue, K. \& Fujita, Y. Nitrogenase Fe protein-like Fe-S cluster is conserved in L-protein $(\mathrm{BchL})$ of dark-operative protochlorophyllide reductase from Rhodobacter capsulatus. FEBS Lett 580, 6151-4 (2006).

16. Nomata, J., Kondo, T., Itoh, S. \& Fujita, Y. Nicotinamide is a specific inhibitor of darkoperative protochlorophyllide oxidoreductase, a nitrogenase-like enzyme, from Rhodobacter capsulatus. FEBS Lett 587, 3142-7 (2013). 
17. Sorlie, M., Chan, J.M., Wang, H., Seefeldt, L.C. \& Parker, V.D. Elucidating thermodynamic parameters for electron transfer proteins using isothermal titration calorimetry: application to the nitrogenase Fe protein. J Biol Inorg Chem 8, 560-566 (2003).

18. Tezcan, F.A., Kaiser, J.T., Howard, J.B. \& Rees, D.C. Structural evidence for asymmetrical nucleotide interactions in nitrogenase. J Am Chem Soc 137, 146-9 (2015).

19. Ryle, M.J., Lanzilotta, W.N. \& Seefeldt, L.C. Elucidating the mechanism of nucleotidedependent changes in the redox potential of the $[4 \mathrm{Fe}-4 \mathrm{~S}]$ cluster in nitrogenase iron protein: the role of phenylalanine 135. Biochemistry 35, 9424-34 (1996).

20. Hosseinzadeh, P. \& Lu, Y. Design and fine-tuning redox potentials of metalloproteins involved in electron transfer in bioenergetics. Biochim Biophys Acta 1857, 557-581 (2016).

21. Liu, J. et al. Metalloproteins containing cytochrome, iron-sulfur, or copper redox centers. Chem Rev 114, 4366-469 (2014).

22. Anthony, J.R., Warczak, K.L. \& Donohue, T.J. A transcriptional response to singlet oxygen, a toxic byproduct of photosynthesis. Proc Natl Acad Sci U S A 102, 6502-7 (2005).

23. Moser, J. \& Brocker, M.J. Methods for nitrogenase-like dark operative protochlorophyllide oxidoreductase. Methods Mol Biol 766, 129-43 (2011).

24. Fujita, Y.\& Bauer, C.E. Reconstitution of light-independent protochlorophyllide reductase from purified bchl and $\mathrm{BchN}-\mathrm{BchB}$ subunits. In vitro confirmation of nitrogenase-like features of a bacteriochlorophyll biosynthesis enzyme. J Biol Chem 275, 23583-8 (2000).

25. Lanz, N.D. et al. RlmN and AtsB as models for the overproduction and characterization of radical SAM proteins. Methods Enzymol 516, 125-52 (2012). 
26. Otwinowski, Z. \& Minor, W. Processing of X-ray diffraction data collected in oscillation mode. Methods Enzymol 276, 307-26 (1997).

27. Winn, M.D. et al. Overview of the CCP4 suite and current developments. Acta Crystallogr D Biol Crystallogr 67, 235-42 (2011).

28. Adams, P.D. et al. PHENIX: a comprehensive Python-based system for macromolecular structure solution. Acta Crystallogr D Biol Crystallogr 66, 213-21 (2010).

29. Emsley, P. \& Cowtan, K. Coot: model-building tools for molecular graphics. Acta Crystallogr D Biol Crystallogr 60, 2126-32 (2004).

30. Stoll, S. \& Schweiger, A. EasySpin, a comprehensive software package for spectral simulation and analysis in EPR. J Magn Reson 178, 42-55 (2006). 


\section{FIGURES \& FIGURE LEGENDS}

a.

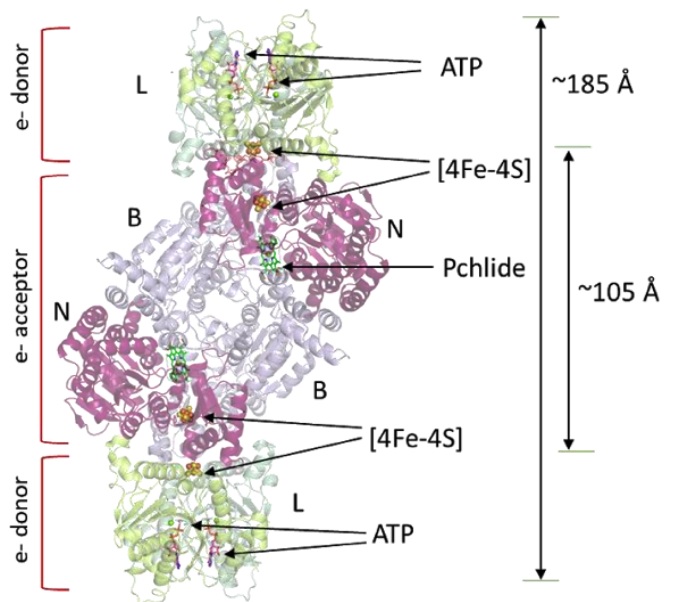

b.

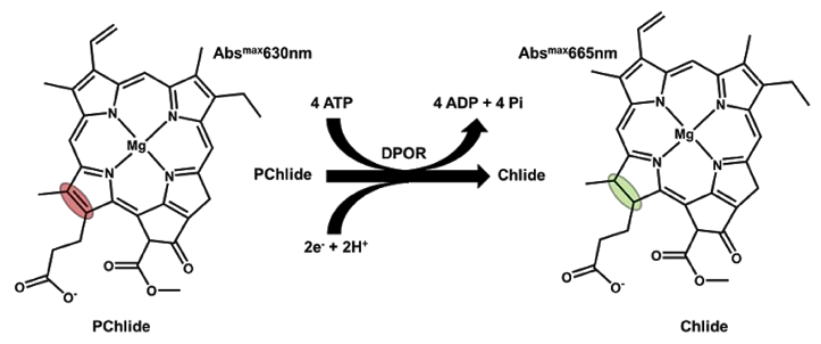

Figure 1. Structure and substrate reduction mechanism of DPOR. (a) Crystal structure of the complete, $\mathrm{ADP}-\mathrm{AlF}_{3}$-stabilized, DPOR complex (PDB:2ynm). BchL subunits are colored green, $\mathrm{BchN}$ is colored purple, and $\mathrm{BchB}$ is violet. The four [4Fe-4S] clusters are shown as spheres, and $\mathrm{ADP}_{-} \mathrm{AlF}_{3}$ and Pchlide are shown as sticks. (b) Schematic of Pchlide reduction to Chlide by DPOR. Two cycles of electron transfer from BchL to BchNB are required for the reduction of the $\mathrm{C} 17=\mathrm{C} 18$ double bond (marked by the colored ovals). 
a.
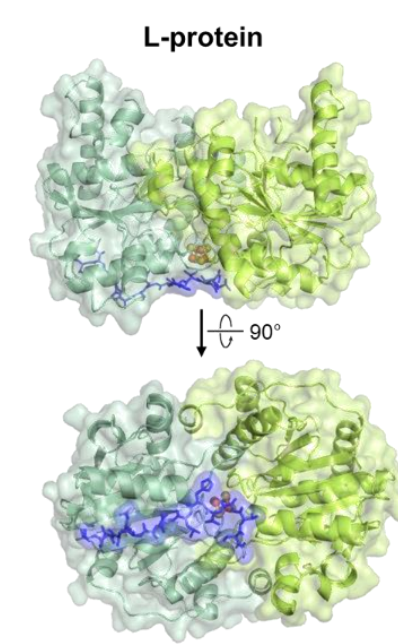

d.

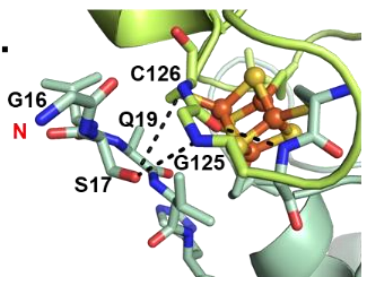

b.
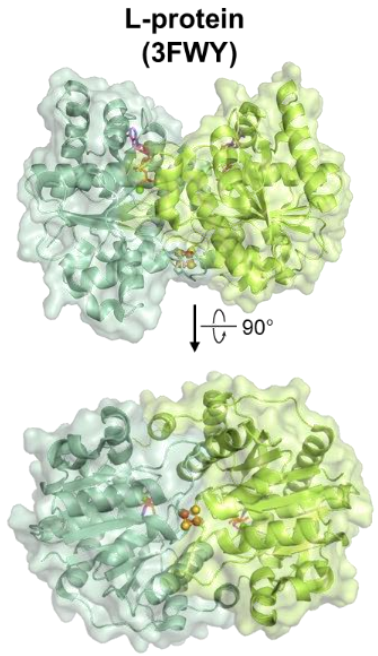

e.

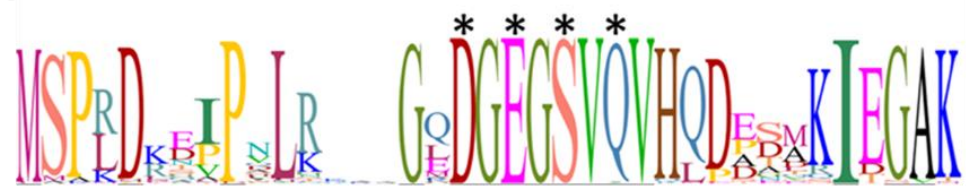

Figure 2. Crystal structure of nucleotide-free $\mathrm{BchL}$ reveals a flexible $\mathrm{N}$-terminal region capping the $[\mathbf{4 F e}-\mathbf{4 S}]$ cluster. Side views (top row) and bottom views (bottom row) of the BchL structure (a) in the absence of nucleotides, (b) with ADP bound (PDB: 2YNM), and (c) in complex with BchNB (PDB: 3FWY). A slight compaction upon the addition of ADP, and a further compaction when in complex with BchNB, can clearly be seen by comparing the side views. The flexible N-terminal region resolved in the nucleotide-free structure (residues 16-29, colored blue in panel a) clearly covers the $[4 \mathrm{Fe}-4 \mathrm{~S}]$ cluster in addition to blocking or directly interacting with several residues predicted to directly interact with BchNB (highlighted in dark purple in panel c). (d) Residues in the flexible $\mathrm{N}$-terminus of chain $\mathrm{C}$ interact with important residues near the [4Fe4S] cluster. The highly conserved Ser17 forms hydrogen bonds with both Cys126 and Gly125 on chain $\mathrm{C}$, the former of which directly interacts with BchNB in the 2YNM structure and the latter additionally interacts with Gly161 on chain D, another predicted BchL-BchNB interface residue. (e) Sequence logo of the N-terminus of BchL generated from alignment of $n=89$ species. Letter height corresponds to the degree of sequence conservation, and the residues mutated in this study are labeled. 

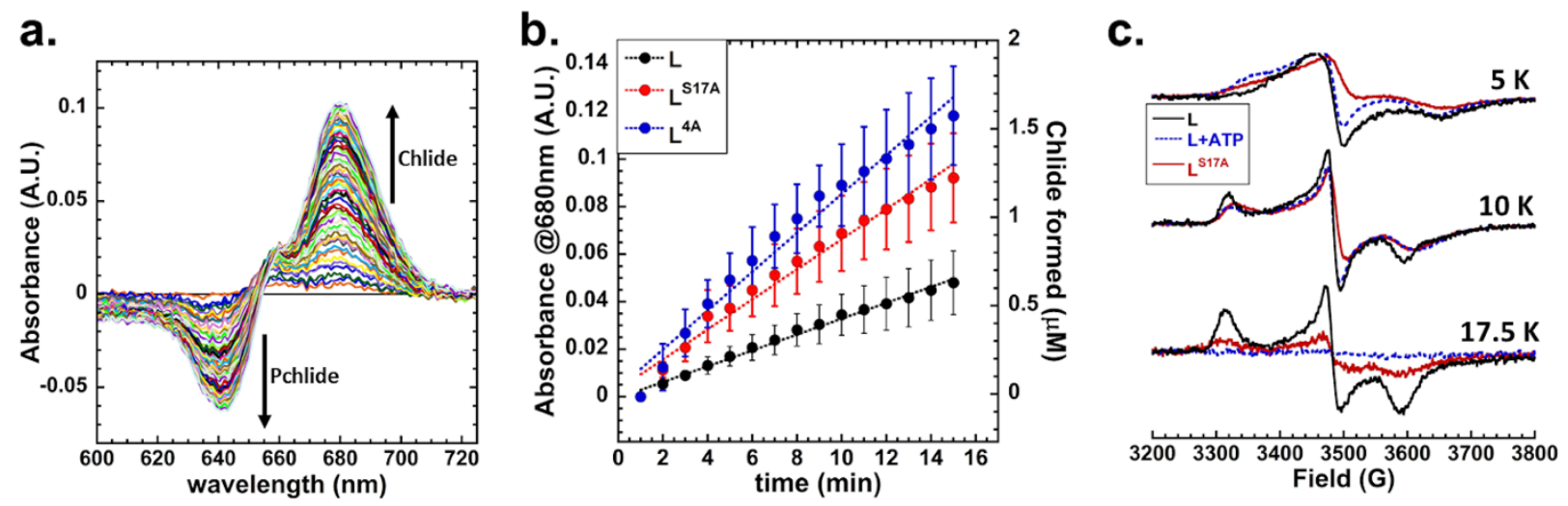

Figure 3. The flexible N-terminal region is auto-inhibitory to BchL function. (a) Representative traces of wild-type reaction in aqueous solution. Arrows represent the trend in absorbance associated with substrate consumption and product formation. (b) Absorbance plot at 680nm (A680) of the in vitro reaction comparing reduction rates as a change in $\mathrm{A}_{680}$ (left axis) and apparent Chlide formation rates (right axis) of BchL (black circles, $k_{o b}=0.044 \pm 0.014 \mu \mathrm{M} . \mathrm{min}^{-1}$ ) $\mathrm{BchL}^{\mathrm{S} 17 \mathrm{~A}}$ (red circles, $k_{o b s}=0.084 \pm 0.019 \mu \mathrm{M} \cdot \mathrm{min}^{-1}$ ), and $\mathrm{BchL}^{4 \mathrm{~A}}$ (blue circles, $k_{\text {obs }}=0.087 \pm 0.016$ $\mu \mathrm{M} . \mathrm{min}^{-1}$ ) (c) EPR spectra comparing BchL (black solid lines) BchL incubated with excess ATP (blue dotted lines), and $\mathrm{BchL}^{\mathrm{S} 17 \mathrm{~A}}$ (Red solid lines) at $5 \mathrm{~K}, 10 \mathrm{~K}$ and $17.5 \mathrm{~K}$ where indicated. 
a.

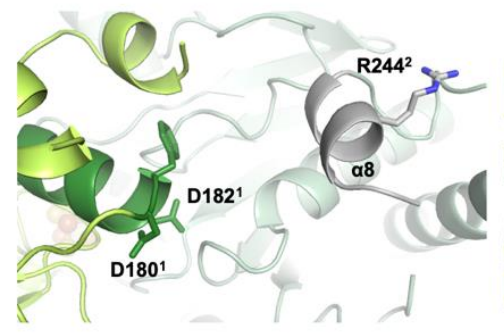

d.

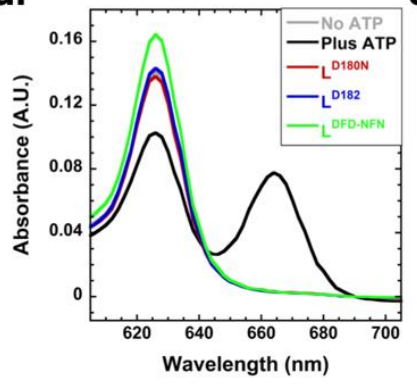

b.

e. 0.6
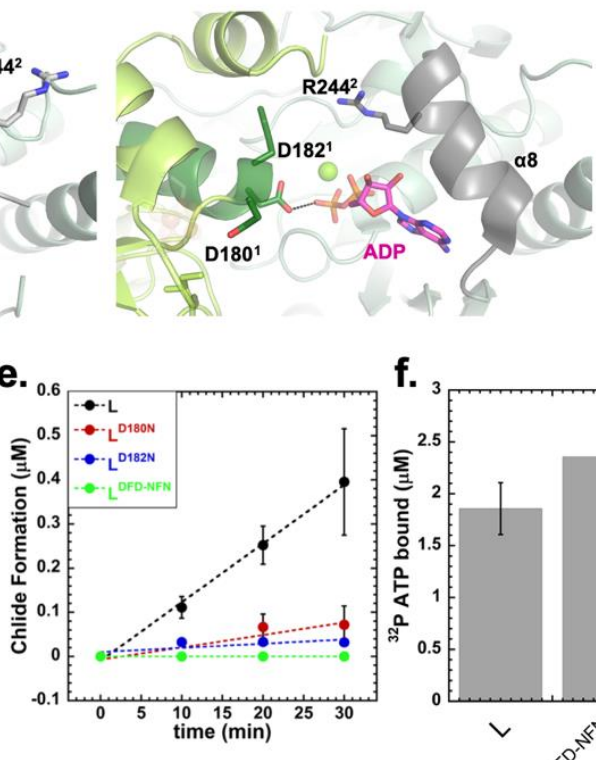

c. f.

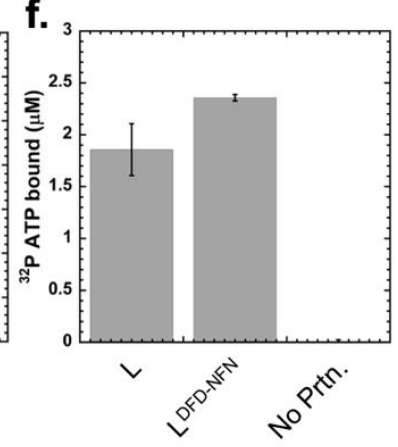

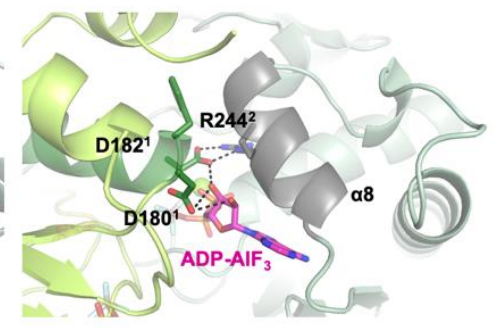

g.

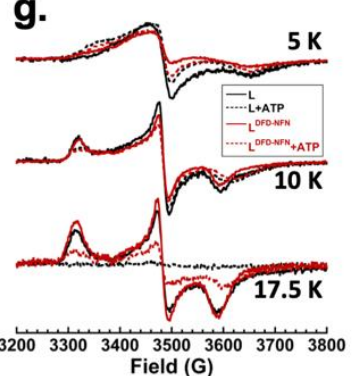

Figure 4. Interactions between ATP and a DFD patch promotes inter-subunit cross stabilization and conformational changes in BchL. Views of the DFD patch (highlighted in green) and associated interactions in three distinct conformations of BchL. (a) In the absence of nucleotides, residues D180 and D182 on chain C form no notable interactions and are a large distance away from residue R244 on the opposing chain D. (b) With ADP bound, the $\alpha 8$-helix, highlighted in dark grey. undergoes considerable motion, bringing R244 much closer to the DFD patch. Additionally, D182 now interacts with the bound ADP. (c) When bound to ADP-AlF 3 and in complex with BchNB, D180 and D182 have extensive interactions with both the bound nucleotide as well inter-subunit interactions with R244. (d) Pchlide reduction activity of BchL and mutant proteins. Chlide formation is monitored as a peak between 660-670 nm and occurs only in the presence of ATP. BchL ${ }^{\mathrm{D} 180 \mathrm{~N}}, \mathrm{BchL}^{\mathrm{D} 182 \mathrm{~N}}$, and $\mathrm{BchL}^{\mathrm{DFD}-\mathrm{NFN}}$, are all defective for substrate reduction. (e) Time course of Chlide formation for reactions containing BchNB, ATP and Pchlide in the presence of various $\mathrm{BchL}$ constructs. $\mathrm{BchL}$ reduces Pchlide to Chlide $\left(k_{o b s} \mathrm{~s}=0.0126 \pm 0065\right.$ $\mu \mathrm{M} \cdot \mathrm{min}^{-1}$ ), and no appreciable Chlide formation is observed for BchL ${ }^{\mathrm{D} 182 \mathrm{~N}}$ or $\mathrm{BchL}{ }^{\mathrm{DFD}-\mathrm{NFN}}$. Severely impaired but detectable reduction activity is observed for $\mathrm{BchL}^{\mathrm{D} 180 \mathrm{~N}}\left(k_{\text {obs }}=0.0028 \pm\right.$ $\left.0.0042 \mu \mathrm{M} \cdot \mathrm{min}^{-1}\right)$. (f) Nitrocellulose filter binding analysis of ATP binding to BchL shows $\sim 2$ ATP bound per BchL dimer to both $\mathrm{BchL}$ and $\mathrm{BchL}{ }^{\text {DFD-NFN }}$, and no non-specific binding to the membranes is observed in the absence of BchL in the reaction. (g) EPR spectra comparing wt BchL (black solid lines), BchL incubated with excess ATP (black dotted lines), BchL ${ }^{\text {DFD-NFN }}{ }^{\text {Red }}$ solid lines), and BchL ${ }^{\text {DFD-NFN }}$ incubated with excess ATP (red dotted lines) at $5 \mathrm{~K}, 10 \mathrm{~K}$, and $17.5 \mathrm{~K}$ as denoted. 
a.

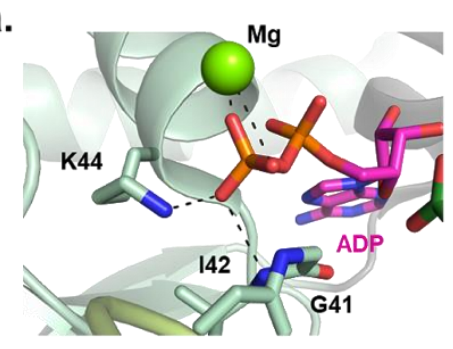

b.

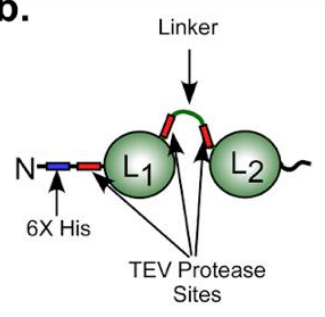

c.

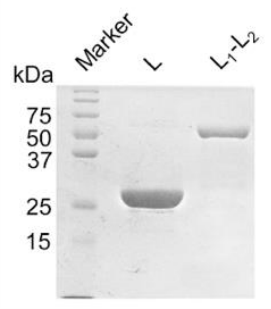

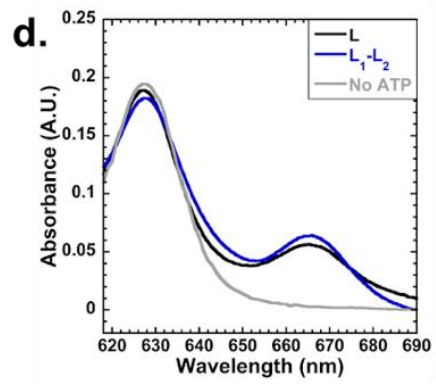
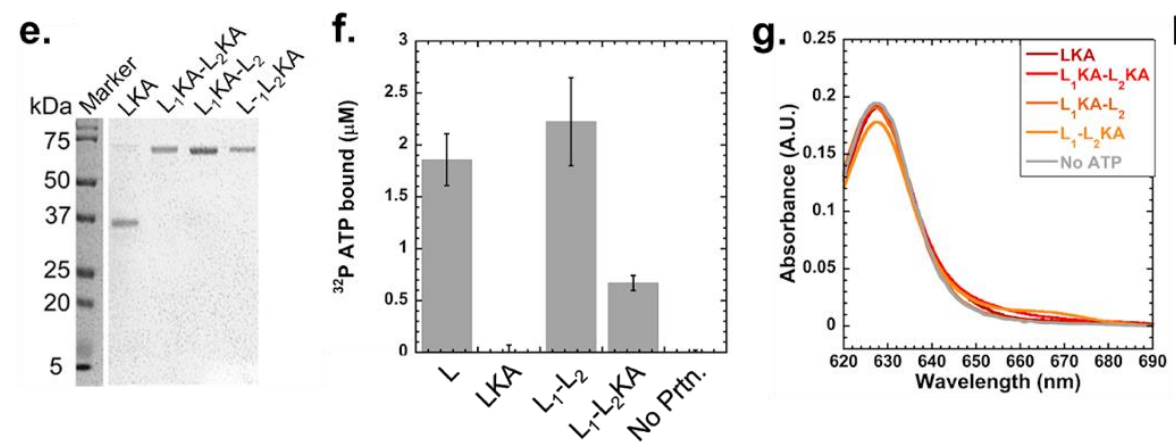

h.

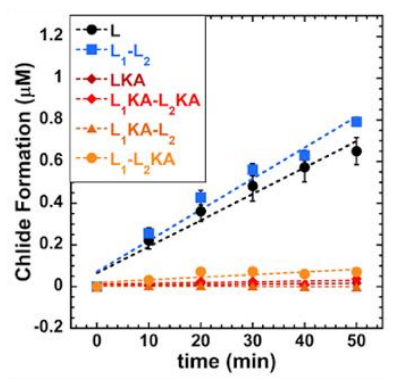

Figure 5. Both ATP binding sites are required for DPOR function. (a) Lys44 stabilizes nucleotide binding through interactions with the phosphate group of ADP (PDB: 3FWY). (b) Schematic of the linked-L-protein design and the positions of the tobacco etch virus (TEV) protease cleavage sites. (c) SDS-PAGE analysis of the purified BchL and linked-BchL-proteins. (d) Spectroscopic analysis of Pchlide reduction activity of BchL (L, black trace), linked-BchLprotein ( $\mathrm{L}_{1}-\mathrm{L}_{2}$, blue trace), and no ATP negative control (No ATP, grey trace). Pchlide absorbance is observed at $625 \mathrm{~nm}$ and Chlide formation is monitored at $665 \mathrm{~nm}$. (e) SDS-PAGE analysis of the purified $\mathrm{BchL}^{\mathrm{K} 44 \mathrm{~A}}$ (LKA) and versions of the single and double Lys44 to Ala substituted linkedBchL-proteins. (f) Nitrocellulose filter binding analysis of ${ }^{32} \mathrm{P}$-ATP binding by wildtype and Lys44 to Ala substituted BchL proteins. $\mathrm{L}$ and $\mathrm{L}_{1}-\mathrm{L}_{2}$ are capable of binding ATP, whereas the singly substituted $\mathrm{L}_{1} \mathrm{KA}-\mathrm{L}_{2}$ is partially able to bind ATP. When both subunits are substituted with Lys44 to Ala, no ATP binding is observed. (g) Linked and unlinked BchL proteins carrying the K44A substitution are incapable of reducing Pchlide. (h) Kinetics of Pchlide reduction measured as a function of Chlide formation is shown. $\mathrm{L}$ and $\mathrm{L}_{1}-\mathrm{L}_{2}$ reduce Pchlide to Chlide $\left(k_{\text {obs }}=0.01266 \pm 0.007\right.$ $\mu \mathrm{M} \cdot \mathrm{min}^{-1}$ and $0.0148 \pm 0.0022 \mu \mathrm{M} \cdot \mathrm{min}^{-1}$, respectively.) 


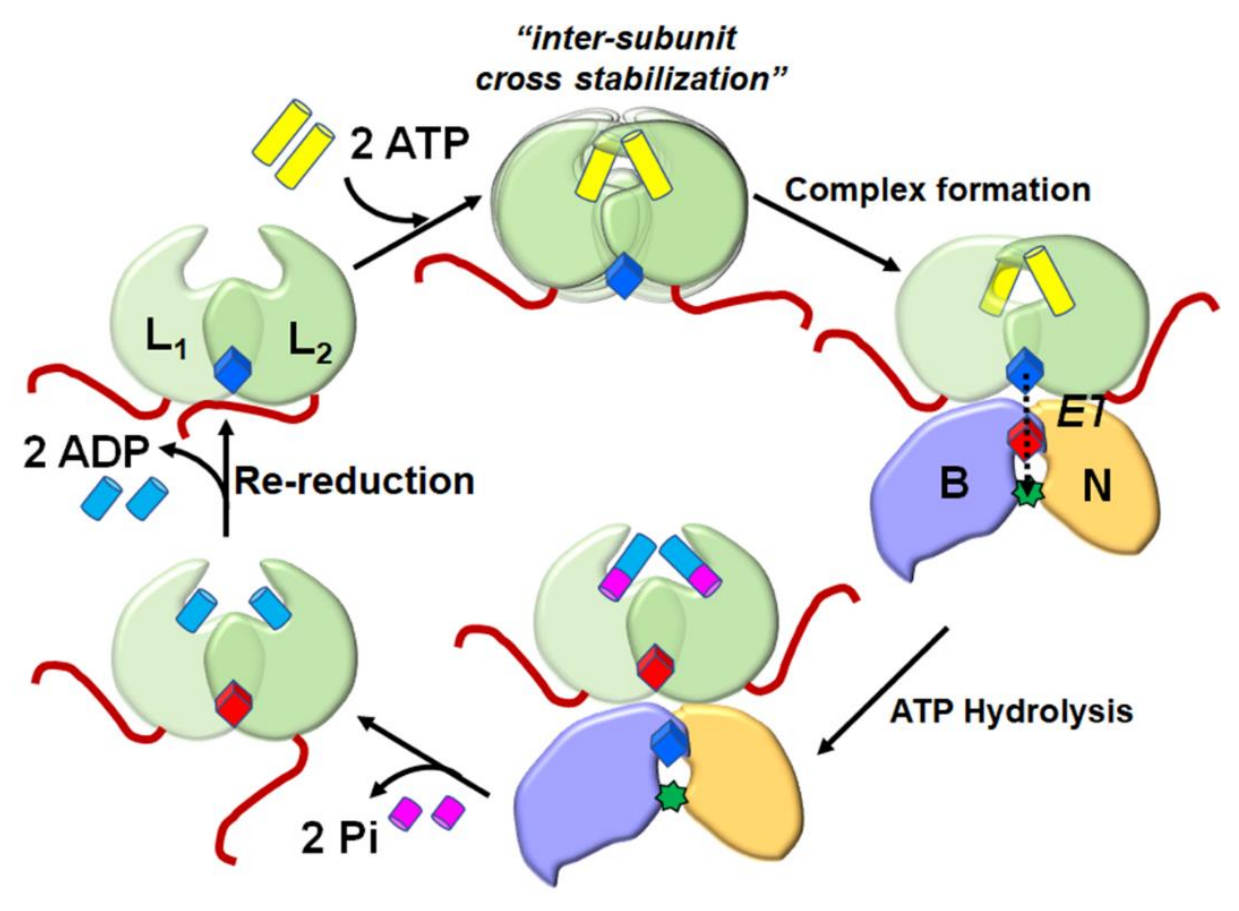

Figure 6. Model for ATP-binding induced release of auto-inhibition by the flexible Nterminus of BchL. Cartoons depict the BchL dimer (green) with one of the two disordered Ntermini binding across the [4Fe-4S] cluster (blue; reduced form). ATP (yellow) binding promotes inter-subunit cross stabilization and associated conformational changes through interactions between the DFD patch and ATP; thereby releasing the flexible N-terminus from the docking surface. Complex formation to the BchNB protein ensues followed by electron transfer (ET), ATP hydrolysis, and product (ADP, Pi) release. The oxidized BchL [4Fe-4S] cluster is depicted in red. The BchL [4Fe-4S] cluster is subsequently re-reduced. 


\section{SUPPLEMENTAL INFORMATION}

\section{Supplementary Table 1: Crystallographic data processing and refinement statistics}

\begin{tabular}{|l|c|}
\hline \multicolumn{2}{|c|}{ Diffraction Statistics } \\
\hline & I121 \\
\hline Space Group & $\mathrm{a}=92.51 \quad \mathrm{~b}=100.92 \quad \mathrm{c}=117.79$ \\
$\alpha=90.00^{\circ} \quad \beta=99.27^{\circ} \quad \gamma=90.00^{\circ}$
\end{tabular}

* Values in parentheses refer to the highest resolution shell 
bioRxiv preprint doi: https://doi.org/10.1101/840439; this version posted November 12,2019 . The copyright holder for this preprint (which was not certified by peer review) is the author/funder, who has granted bioRxiv a license to display the preprint in perpetuity. It is made available under aCC-BY-NC-ND 4.0 International license.

Supplemental Table 2. Oligonucleotide primers used in this study

\begin{tabular}{|l|l|l|}
\hline 1 & $\begin{array}{l}\text { L1 } \\
\text { monomer } \\
\text { R }\end{array}$ & $\begin{array}{l}5^{\prime} \\
\text { ATTATTCATATGGTCGACTGATTGGAAGTATAGATTCTCTGCGGCCGCTCCATCGAA } \\
\text { ACCCAGCAACTCGAAATTTCGCGATCCGGCAGCG 3' }\end{array}$ \\
\hline 2 & $\begin{array}{l}\text { ACYCDu } \\
\text { etUp1 }\end{array}$ & $5^{\prime}$ GGATCTCGACGCTCTCCCT 3' \\
\hline 3 & $\begin{array}{l}\text { L2 } \\
\text { monomer }\end{array}$ & $\begin{array}{l}5^{\prime} \\
\text { AATAATCATATGGAGAATCTATACTTCCAATCAAAGCTTATGAGCCCGAAAGACTTA } \\
\text { F ATA CCG ACC GGA GCG GAT GGCGAGGGCTCGGTCCAGGTGC 3' }\end{array}$ \\
\hline
\end{tabular}




\section{Supplemental Methods}

Construction of $L_{1}-L_{2}$ linked-L-protein constructs: We used a previously described BchL-RSFDuet 1 plasmid backbone for cloning the linked-BchL construct. ${ }^{1}$ The two consecutive BchL ORFs are designated $\mathrm{L}_{1}$ and $\mathrm{L}_{2}$, respectively. For construct 1 - 'BchL 1 -pRSF-Duet1', the ORF encoding the $\mathrm{L}_{1}$ monomer was PCR amplified using primers \#1 and \#2 (Supplemental Table 2), designed to eliminate the stop codon at the end of the BchL ORF and insert a TEV protease recognition sequence. The PCR product was engineered into an empty RSF-Duet 1 plasmid using BamHI and NdeI. For construct 2 - 'BchL 2 -pRSF-Duet1', the ORF encoding the $\mathrm{L}_{2}$ monomer was similarly PCR amplified using primer \#3 (Supplemental Table 2) and a T7 reverse primer and engineered into an empty pRSF Duet1 plasmid using Nde1 and BamHI. This ORF encodes the linker between the $\mathrm{L}_{1}$ and $\mathrm{L}_{2}$ genes, as well as a second TEV protease recognition sequence. The flanking TEV sites were designed to remove the intervening linker, post-purification with TEV protease, if necessary. For construct 3 - 'BchL $\mathrm{B}_{1}-\mathrm{L}_{2}$-pRSF-Duet1', the $\mathrm{L}_{2}$ ORF was cut from BchL 2 -pRSFDuet1 with NdeI and BamHI and engineered into BchL 1 -pRSF-Duet1 using the same restriction sites. Lys44 to Ala substitutions were first introduced into the respective BchL 1 -pRSF-Duet1 or BchL2-pRSF-Duet1 plasmids using Q5 mutagenesis and subsequently subcloned into the BchL ${ }^{-}$ $\mathrm{L}_{2}$-pRSF-Duet1 plasmid as described above. The subsequent iterations of the linked L constructs (Supplemental Fig. S4c), where the linker lengths were varied, were synthesized as codonoptimized synthetic genes (Genscript Inc.). 


\section{Supplemental Figure 1}
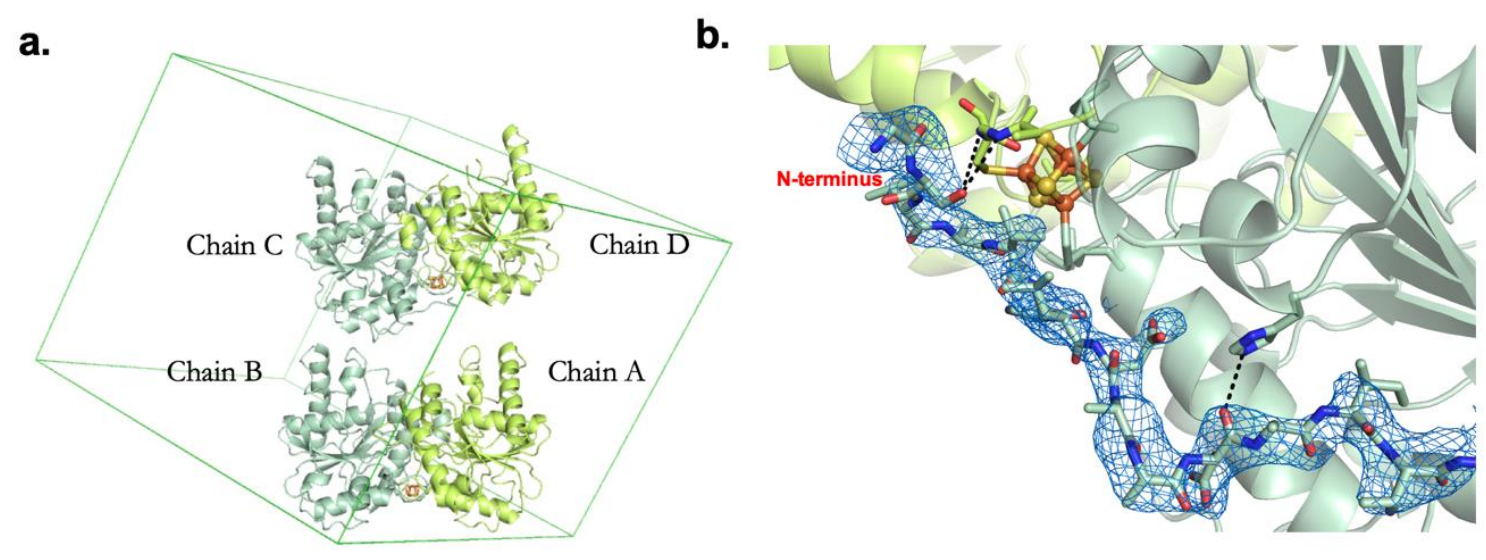

Supplementary Figure 1: Crystal structure of nucleotide-free BchL a. The asymmetric unit of the crystal structure comprises two dimers of BchL chains. The unit cell is outlined in green. $\mathbf{b}$. Fo-Fc map of density for the previously unresolved $\mathrm{N}$-terminal tail in chain $\mathrm{C}$ (shown as blue mesh, contoured at $1.0 \sigma$ ). Clear, continuous density is visible for the backbone of the entire tail up to residue 16. Interactions of tail residue Ser17 with residues Gly125 and Cys126 on chain D as well as the hydrogen-bonding interaction between Asp26 and His169 of chain C are highlighted. 


\section{Supplemental Figure 2}

a.

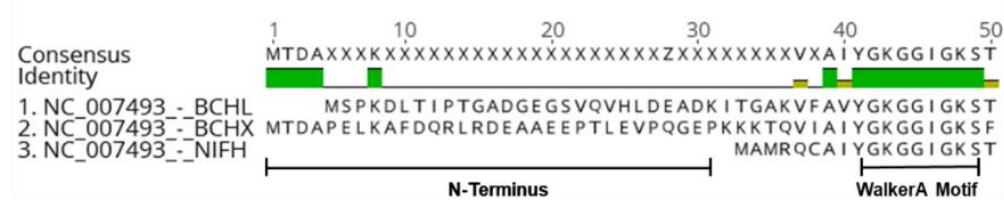

b. BchL N-terminus Conservation

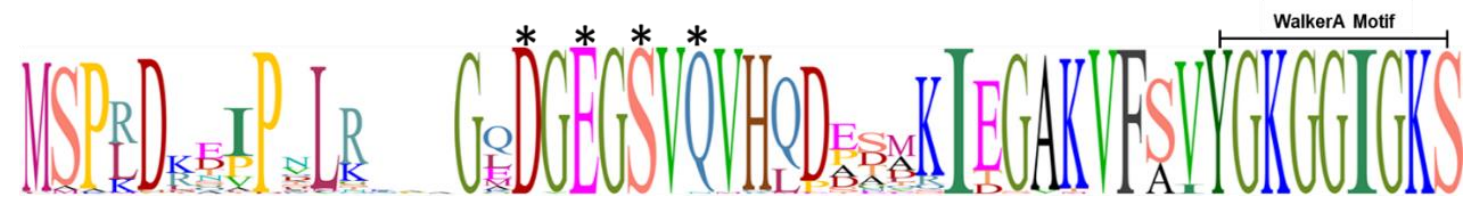

C. BchX N-terminus Conservation

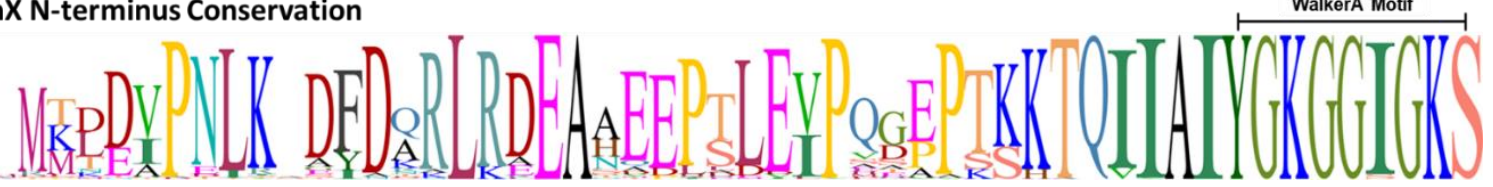

Supplementary Figure 2: a. Sequence alignment BchL, BchX, and NifH (the Nitrogenase Feprotein). N-terminus length highlighted for $\mathrm{BchL}$ and $\mathrm{BchX}$. Walker A motif sequence conservation is also labeled. b. Sequence logo of the N-terminus of BchL generated from alignment of $n=89$ species. Letter height represents sequence conservation. Critical amino acids for capping and generation of $\mathrm{BchL}^{\mathrm{S} 17 \mathrm{~A}}$ and $\mathrm{BchL}^{4 \mathrm{~A}}$ are labeled with asterisks. c. Sequence logo of the N-terminus of $\mathrm{BchX}$ generated from alignment of $n=89$ species. Letter height represents sequence conservation. Figure demonstrates lack of conservation in N-terminus of BchX. NifH Nterminus conservation is not shown as it lacks enough residues to potentially cap its cluster. 


\section{Supplemental Figure 3}

a.

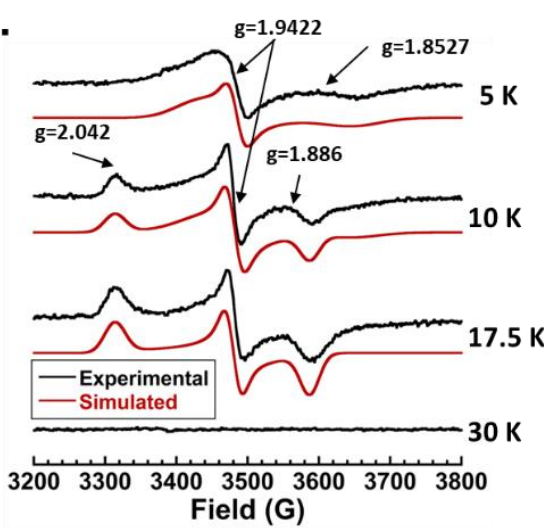

d.

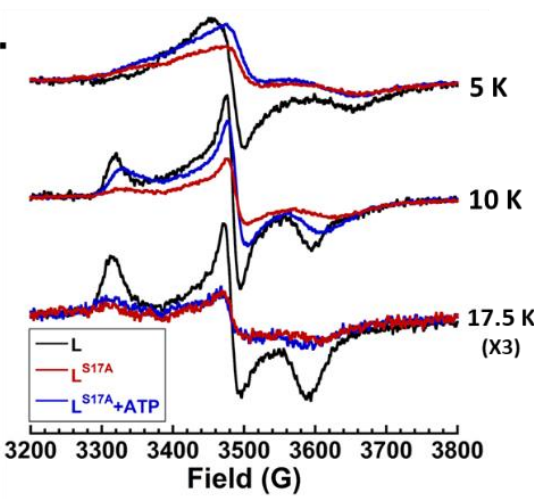

b.

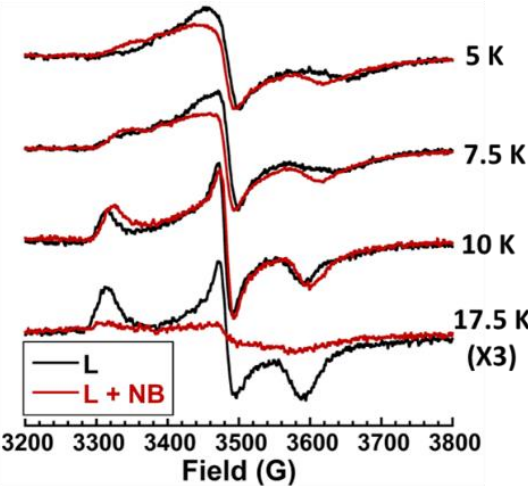

c.

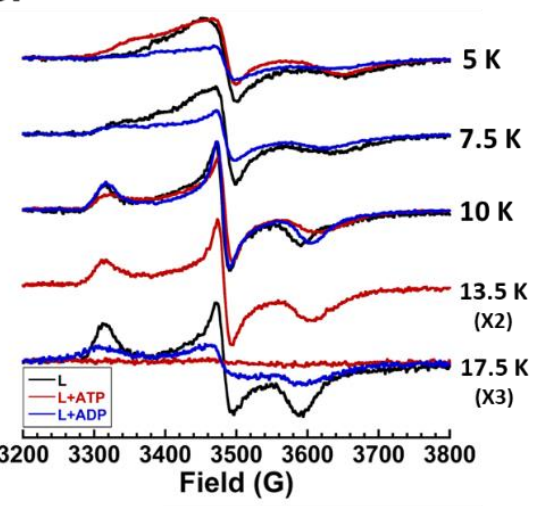

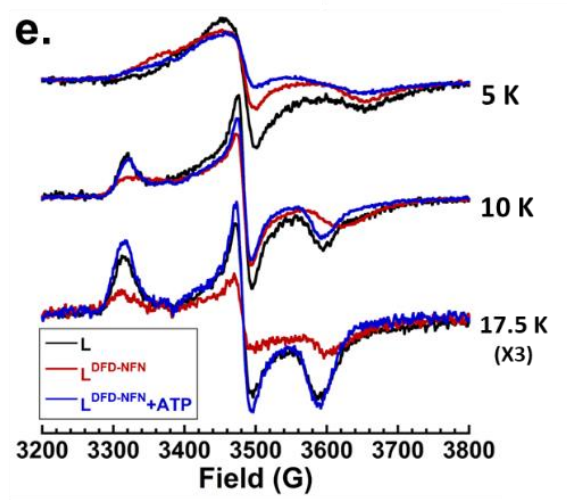

Supplementary Figure 3: a. Experimentally determined (black) and simulated (red) EPR spectra of BchL at $5 \mathrm{~K}, 10 \mathrm{~K}, 17.5 \mathrm{~K}$, and $30 \mathrm{~K}$. No absorbance was detected at 35K, typical of [4Fe-4S] clusters. There appear to be two species, a fast-relaxing almost-axial species $\left(\mathrm{g}=\left[\begin{array}{l}1.9750 \\ 1.9422\end{array}\right.\right.$ 1.8527]) and a slower-relaxing rhombic species $(\mathrm{g}=[2.04201 .94421 .8860])$. The spectrum at 10 $\mathrm{K}$ was simulated using a $40 \%$ axial:60 \% rhombic mixture. The g-values for the axial signal are highly unusual in having all three values less than 2.0. In addition, the line shape could not be replicated well, probably due to rapid passage artifacts. The rhombic signal, on the other hand, is "well-behaved". b. EPR spectra of BchL (black traces) and BchL with 1:1 BchNB (red traces) at indicated temperatures. Upon adding BchNB, there are spectral and relaxation changes. The highfield resonance of the axial signal occurs at a higher g-value (lower field) and features due to the g1 of the rhombic signal persist (distorted by rapid passage) at $5 \mathrm{~K}$. The rhombic signal is very similar in shape to that of the BchL protein alone, but in the presence of $\mathrm{BchNB}$, the signal undergoes significant relaxation broadening at $17.5 \mathrm{~K}$. c. EPR spectra of BchL (black traces), BchL incubated with excess ATP (red traces), and BchL incubated with excess ADP (blue traces) at indicated temperatures. ATP incubation produced the most drastic spectral and relaxation changes, particularly at higher temperatures, broadening relaxation almost entirely. ADP incubations produced similar, though not identical effects as BchNB incubation. d. EPR spectra of BchL (black traces), BchL ${ }^{\mathrm{S} 17 \mathrm{~A}}$ (red traces), and $\mathrm{BchL}^{\mathrm{S} 17 \mathrm{~A}}$ incubated with excess ATP (blue races) at indicated temperatures. The slow relaxing axial species was distorted for $\mathrm{BchL}^{\mathrm{S} 17 \mathrm{~A}}$ compared to wildtype. 
The fast relaxing species was significantly broadened compared to wild-type and was unaffected by ATP incubation, though the mixed-species signal and axial species were both significantly diminished compared to wt. e. EPR spectra of BchL (black traces), BchL ${ }^{\text {DFD-NFN }}$ (red traces), and BchL ${ }^{\text {DFD-NFN }}$ incubated with excess ATP (blue traces). BchL ${ }^{\text {DFD-NFN }}$ produced spectra similar to wt BchL with some minor distortions in the axial signal. ATP incubation with BchL ${ }^{\text {DFD-NFN }}$ produced spectral changes similar to those produced for the wildtype BchL, though to a lesser extent. 
a.

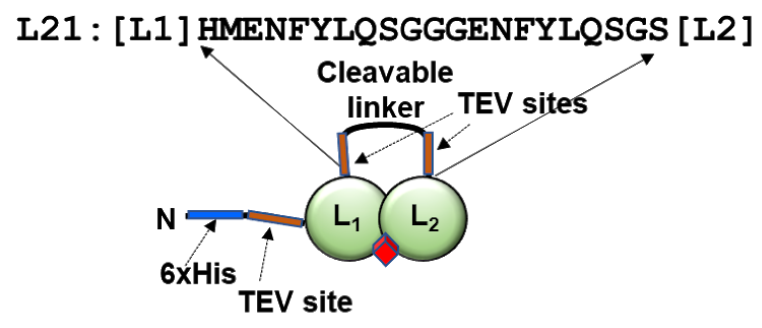

C.

L5: [L1]VDGGGGGSM [L2]

L10: [L1] VDGGGGGGGGGGSM [L2]

L15: [L1] VDGGGGGGGGGGGGGGSM [L2]

L20 : [L1] VDGGGGGGGGGGGGGGGGGGGSM [L2]

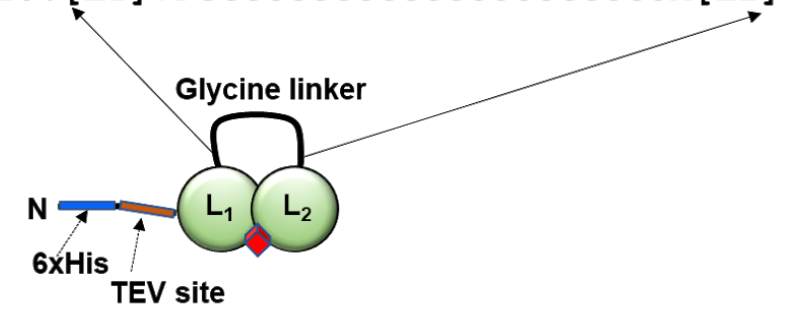

b.
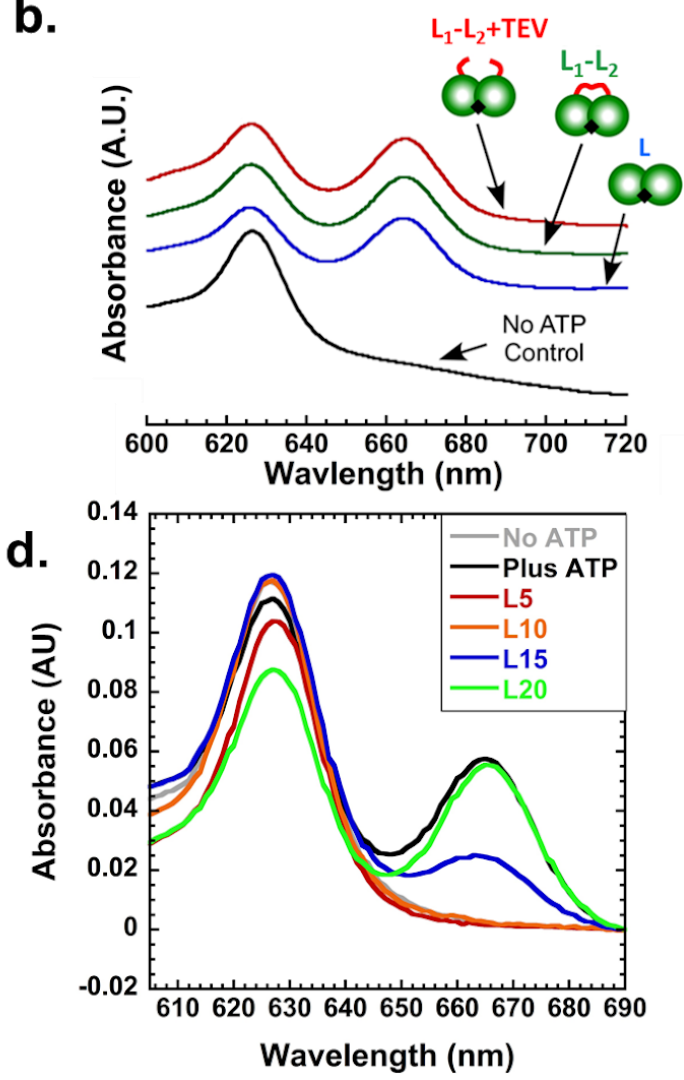

Supplementary Figure 4: a. Cartoon representation of the generated cleavable linked BchL construct, and the amino acid sequence of the linker. b. Absorbance plot of acetone extraction of pigments after in vitro reaction. Cartoon representations of constructs are shown, and absorbance traces are shifted for clarity. The no ATP (negative control -black), WT reaction (positive control -blue), linked WT (green), and linked WT after cleavage with TEV protease (red) are shown. c. Cartoon representation of poly-glycine linkers lacking protease sites, with amino acid sequences of various linker lengths. d. Absorbance plot of acetone extraction of pigments after in vitro reaction. The no ATP negative control and WT reaction positive control shown as grey and black traces respectively. L5, L10, L15, and L20 construct traces are shown as red, orange, blue, and green lines, respectively. 


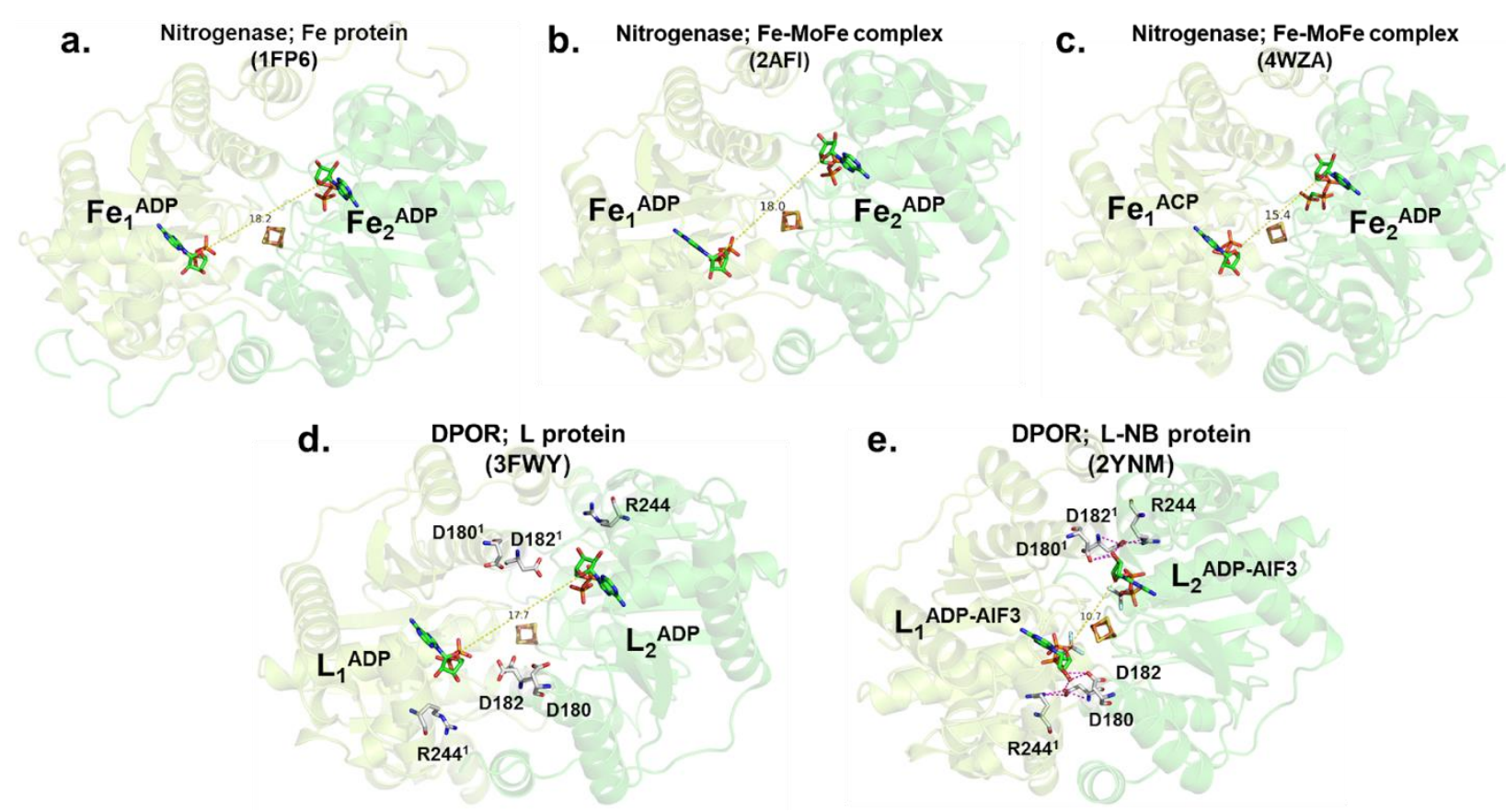

Supplementary Figure 5. Crystal structures of substrate-bound BchL and the Fe-protein from Nitrogenase. Monomers are colored light and dark green as in previous figures, with highlighted residues and [4Fe-4S] clusters shown as sticks. Distance measurements are shown as dotted yellow lines. a. ADP-bound Fe-protein. b. ADP bound Fe-protein in complex with Mo-Fe protein. c. ADP and ACP bound Fe-protein in complex with MoFe protein. d. ADP bound BchL. e. ADP-AlF3 bound DPOR in complex with BchNB (not shown). 
a.

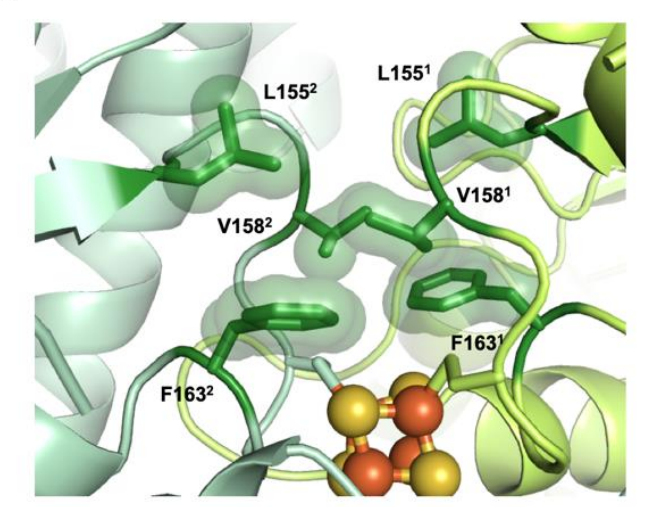

b.

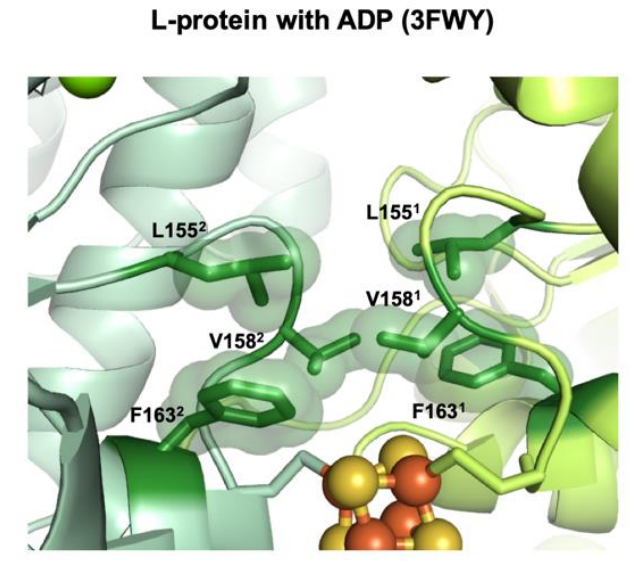

Supplementary Figure 6: Perturbations in the Switch-II region change the local environment around the $[4 \mathrm{Fe}-4 \mathrm{~S}]$ cluster. Highlighted in dark green are the interactions of six hydrophobic residues, Leu155, Val158 and Phe163 on each chain, in the absence of nucleotide a. and presence of ADP b. The environment directly above the [4Fe-4S] cluster becomes less hydrophobic upon the addition of ADP, largely as a result of repositioning of the adjacent Phe163 residues relative to the cluster. More hydrophobic environments generally correlate with increased reduction potential for redox-active metal centers, suggesting that the binding of Mg-ADP acts as a form of redox control in BchL by increasing the tendency of the cluster to become oxidized.

\section{Supplemental References}

1. Corless, E.I., Mettert, E.L., Kiley, P.J. \& Antony, E. Elevated Expression of a Functional Suf Pathway in E. coli BL21(DE3) Enhances Recombinant Production of an Iron-Sulfur Cluster Protein. Journal of Bacteriology, JB.00496-19 (2019). 